\title{
Mechanisms of SARS-CoV-2 entry into cells
}

Cody B. Jackson (10 ${ }^{1,4}$, Michael Farzan $\left(\mathbb{1}^{1}\right.$, Bing Chen $\mathbb{1}^{2,3 凶}$ and Hyeryun Choe $\mathbb{1}^{1 凶}$

Abstract | The unprecedented public health and economic impact of the COVID-19 pandemic caused by infection with severe acute respiratory syndrome coronavirus 2 (SARS-CoV-2) has been met with an equally unprecedented scientific response. Much of this response has focused, appropriately, on the mechanisms of SARS-CoV-2 entry into host cells, and in particular the binding of the spike (S) protein to its receptor, angiotensin-converting enzyme 2 (ACE2), and subsequent membrane fusion. This Review provides the structural and cellular foundations for understanding the multistep SARS-CoV-2 entry process, including S protein synthesis, $S$ protein structure, conformational transitions necessary for association of the $S$ protein with ACE2, engagement of the receptor-binding domain of the $S$ protein with ACE2, proteolytic activation of the $\mathrm{S}$ protein, endocytosis and membrane fusion. We define the roles of furin-like proteases, transmembrane protease, serine 2 (TMPRSS2) and cathepsin L in these processes, and delineate the features of ACE2 orthologues in reservoir animal species and S protein adaptations that facilitate efficient human transmission. We also examine the utility of vaccines, antibodies and other potential therapeutics targeting SARS-CoV-2 entry mechanisms. Finally, we present key outstanding questions associated with this critical process.

'Department of Immunology and Microbiology, Scripps Research, Jupiter, FL, USA.

${ }^{2}$ Division of Molecular Medicine, Boston Children's Hospital, Boston, MA, USA.

${ }^{3}$ Department of Pediatrics, Harvard Medical School, Boston, MA, USA.

${ }^{4}$ Present address: Charles $E$. Schmidt College of Medicine, Florida Atlantic University, Boca Raton, FL, USA.

$\otimes_{e-m a i l:}$

bchen@crystal.harvard.edu; hchoe@scripps.edu

https://doi.org/10.1038/ s41580-021-00418-x
Severe acute respiratory syndrome coronavirus 2 (SARS-CoV-2) is the causative agent of COVID-19, which escalated into a global pandemic in 2020 . SARS-CoV-2 is an enveloped, positive-sense, singlestranded RNA virus of the genus Betacoronavirus ${ }^{1}$. In addition to SARS-CoV, which was responsible for the 2002-2004 SARS epidemic and which shares 79\% nucleotide sequence identity with SARS-CoV-2 (REF. $\left.{ }^{1}\right)$, the genus also includes human coronavirus ( $\mathrm{HCoV})-\mathrm{OC} 43$, HCoV-HKU1 and Middle East respiratory syndrome coronavirus (MERS-CoV). SARS-CoV-2 relies on its obligate receptor, angiotensin-converting enzyme 2 (ACE2), to enter cells ${ }^{2-6}$; ACE2 was originally identified in 2003 as the receptor for SARS-CoV ${ }^{7}$. ACE2 is also the receptor for alphacoronavirus HCoV-NL63, which, together with another alphacoronavirus, $\mathrm{HCoV}-229 \mathrm{E}$, and betacoronaviruses $\mathrm{HCoV}-\mathrm{OC} 43$ and $\mathrm{HCoV}-\mathrm{HKU} 1$, is a known causative agent of mild upper respiratory tract infections ${ }^{8}$.

The coronavirus virion is made up of the nucleocapsid (N), membrane (M), envelope (E) and spike (S) proteins, which are structural proteins (FIG. 1). The entry steps of the viral particles - encompassing attachment to the host cell membrane and fusion - are mediated by the $\mathrm{S}$ glycoprotein. S protein is assembled as a homotrimer and is inserted in multiple copies into the membrane of the virion giving it its crown-like appearance. Entry glycoproteins of many viruses, including HIV-1, Ebola virus and avian influenza viruses, are cleaved into two subunits - extracellular and transmembrane - in the infected cells (that is, the cleavage occurs before release of the virus from the cell that produces it). Similarly, the S protein of some coronaviruses is cleaved into S1 and S2 subunits during their biosynthesis in the infected cells, while the $S$ protein of other coronaviruses is cleaved only when they reach the next target cell. SARS-CoV-2, like MERS-CoV, belongs to the first category: its $\mathrm{S}$ protein is cleaved by proprotein convertases such as furin in the virus-producer cells ${ }^{9,10}$ (FIG. 1). Therefore, the $S$ protein on the mature virion consists of two non-covalently associated subunits: the S1 subunit binds ACE2 and the S2 subunit anchors the S protein to the membrane. The S2 subunit also includes a fusion peptide and other machinery necessary to mediate membrane fusion upon infection of a new cell ${ }^{11}$.

Receptor engagement by viral entry glycoproteins, typically with other triggers, induces dramatic conformational changes in both subunits that bring the viral and cellular membranes together, ultimately creating a fusion pore that allows the viral genome to reach the cell cytoplasm. For SARS-CoV-2, one such trigger is the cleavage of an additional site internal to the $\mathrm{S} 2$ subunit, termed the 'S2' site'. ACE2 engagement by the virus exposes the S2' site. S2' site cleavage - by transmembrane protease, serine 2 


\section{Angiotensin-converting enzyme 2 \\ (ACE2). A zinc \\ metalloproteinase that cleaves angiotensin I and angiotensin II into angiotensin-(1-9) and angiotensin-( $1-7)$ as well as other peptide substrates, countering vasoconstriction mediated by angiotensin- converting enzyme (ACE) It also serves as the primary receptor for severe acute respiratory syndrome coronavirus (SARS-CoV) SARS-CoV-2 and other sarbecoviruses.}

Proprotein convertases A family of membraneassociated or secreted serine proteases involved in activating proproteins, including prohormones, by removing the blocking portion of the protein

Furin

A proprotein convertase family transmembrane protein that resides in the Golgi apparatus. It recognizes and cleaves the polybasic motifs of various viral entry glycoproteins, including those of HIV-1, avian influenza A virus and severe acute respiratory syndrome coronavirus 2

\section{Fusion peptide}

A hydrophobic peptide of 20-25 residues that mediates fusion between viral and cellular membranes. The viral entry process requires appropriately timed exposure of this peptide, typically achieved through proteolysis and conformational changes in the entry glycoprotein after receptor binding.

Transmembrane protease, serine 2

(TMPRSS2). A transmembrane serine protein with broad specificity and multiple potential cellular substrates. It is

frequently utilized by respiratory viruses, including severe acute respiratory syndrome coronavirus 2 , to activate their entry glycoproteins.

\section{Cathepsin L}

A soluble cysteine proteinase involved in protein degradation and antigen processing in the late endosomes and

lysosomes. Several viruses, including Ebola virus and severe acute respiratory syndrome coronavirus, utilize this enzyme to activate their entry glycoproteins.

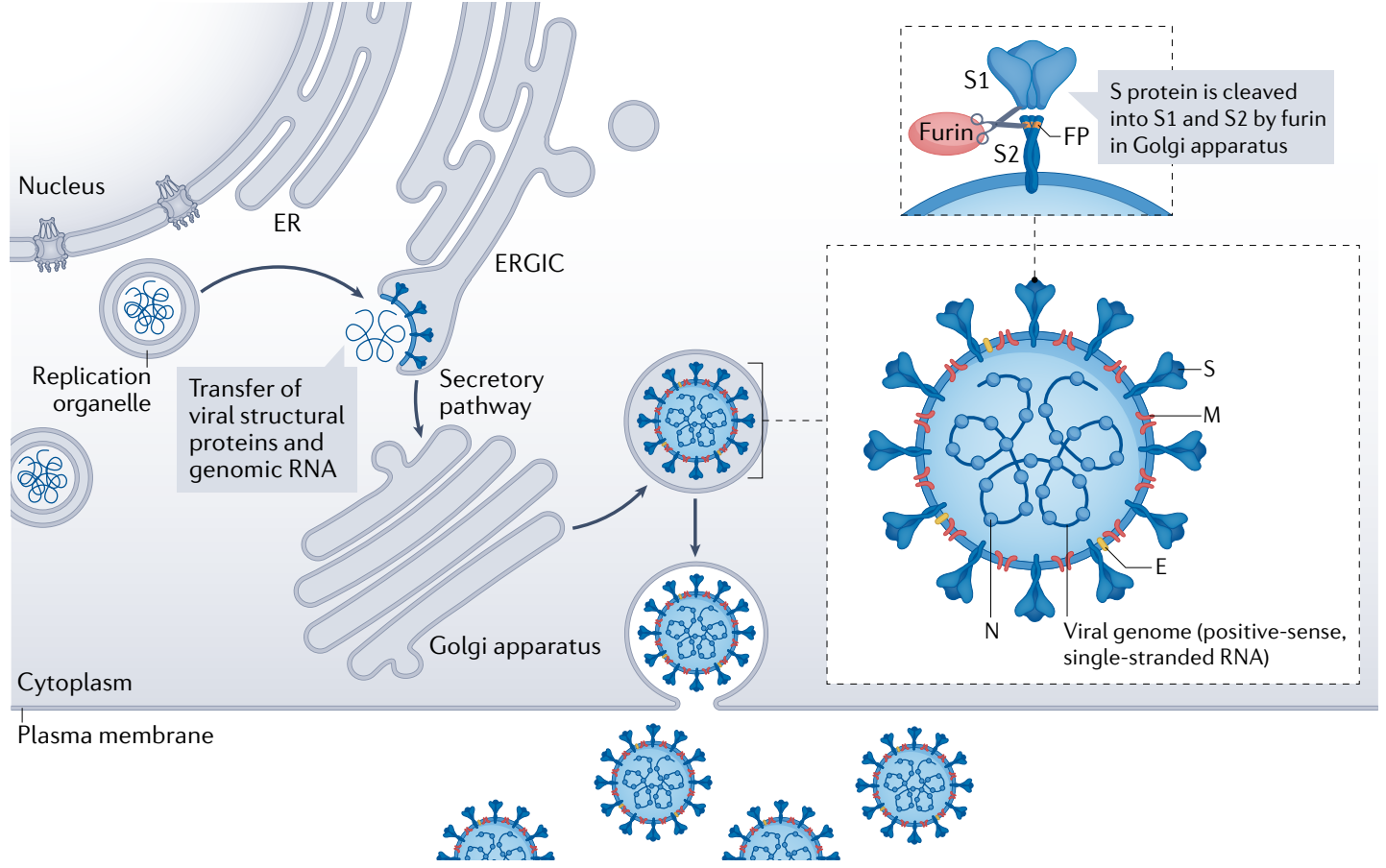

Fig. 1 | Coronavirus structure and maturation. Infection by a coronavirus induces in the perinuclear area the formation of new membranous structures of various sizes and shapes, which as a whole are referred to as 'replication organelles"225-229. These structures - observed by electron microscopy in cells infected with mouse hepatitis virus, severe acute respiratory syndrome coronavirus or Middle East respiratory syndrome coronavirus and typically surrounded by double membranes likely originate from the endoplasmic reticulum (ER) and house viral replication complexes, sequestering them from cellular innate immune molecules. Viral structural proteins and genomic RNA synthesized at the replication site are then translocated through an unknown mechanism to the ER-Golgi intermediate compartment (ERGIC), where virus assembly and budding occur $^{228,230}$. Only four viral proteins - the spike (S), envelope (E), membrane (M) and nucleocapsid (N) proteins - are incorporated into the virion. While the $\mathrm{N}$ protein bound to the viral genomic RNA is packed inside the virion, the structural proteins $\mathrm{S}, \mathrm{E}$ and $\mathrm{M}$ are incorporated in the virion membrane. The $\mathrm{S}$ protein, assembled as a trimer, giving the appearance of a crown (corona), mediates major entry steps, including receptor binding and membrane fusion. During biosynthesis and maturation in the infected cell, the $\mathrm{S}$ protein is cleaved by furin or furin-like proprotein convertase in the Golgi apparatus into the S1 and S2 subunits, which remain associated ${ }^{9,10}$. The S protein on the virus therefore consists of two non-covalently associated subunits with different functions: in the new target cell, the S1 subunit binds the receptor and the $\mathrm{S} 2$ subunit anchors the $\mathrm{S}$ protein to the virion membrane and mediates membrane fusion. The $\mathrm{E}$ and $\mathrm{M}$ proteins contribute to virus assembly and budding through the interactions with other viral proteins ${ }^{231,232}$. Assembled viruses bud into the ERGIC lumen and reach the plasma membrane via the secretory pathway, where they are released into the extracellular space after virus-containing vesicles fuse with the plasma membrane. FP, fusion peptide.

(TMPRSS2) $)^{12-14}$ at the cell surface or by cathepsin $L^{15,16}$ in the endosomal compartment following ACE2-mediated endocytosis ${ }^{17,18}$ - releases the fusion peptide, initiating fusion pore formation (FIG. 1). Because the viral genome must access the cytoplasm and because it can do so only as this pore expands and the viral and cell membranes are seamlessly combined, every step of this process is important.

This Review provides the structural and cell biological foundations for understanding the multistep SARS-CoV-2 entry process. Much of the SARS-CoV-2 entry process was informed by prior studies of SARS-CoV, which shares a similar entry process, and combined interpretation of studies focused on infectious SARS-CoV-2, pseudoviruses, virus-like particles and structural analyses of the $S$ protein. We also discuss $S$ protein evolution and mutation supporting adaptation to the human host and provide an overview of current vaccine approaches and therapeutic strategies targeting SARS-CoV-2 entry mechanisms.

\section{Coronavirus S protein}

The monomeric $\mathrm{S}$ protein of SARS-CoV-2 is a type I membrane protein with $66 \mathrm{~N}$-linked glycans per $S$ protein trimer ${ }^{19}$ and belongs to the so-called class I viral fusion proteins exemplified by the influenza virus haemagglutinin protein. Accordingly, $\mathrm{S}$ proteins of coronaviruses and haemagglutinin share structural organization and conformational transition that promotes membrane fusion ${ }^{20}$.

Overall structure of $S$ trimer. Structural biology of the SARS-CoV-2 $S$ protein has advanced very rapidly since the initial outbreak of COVID-19. Structures of $S$ protein fragments (FIG. 2a) derived from the Wuhan-Hu-1 strain, including the ectodomain stabilized in its prefusion conformation $^{4,21,22}$, receptor binding domain (RBD)ACE2 complexes ${ }^{2,5,6,23}$ and segments of the S2 subunit in the postfusion conformation ${ }^{24}$, were determined by either cryo-electron microscopy (cryo-EM) or X-ray crystallography. They were followed by structures of 

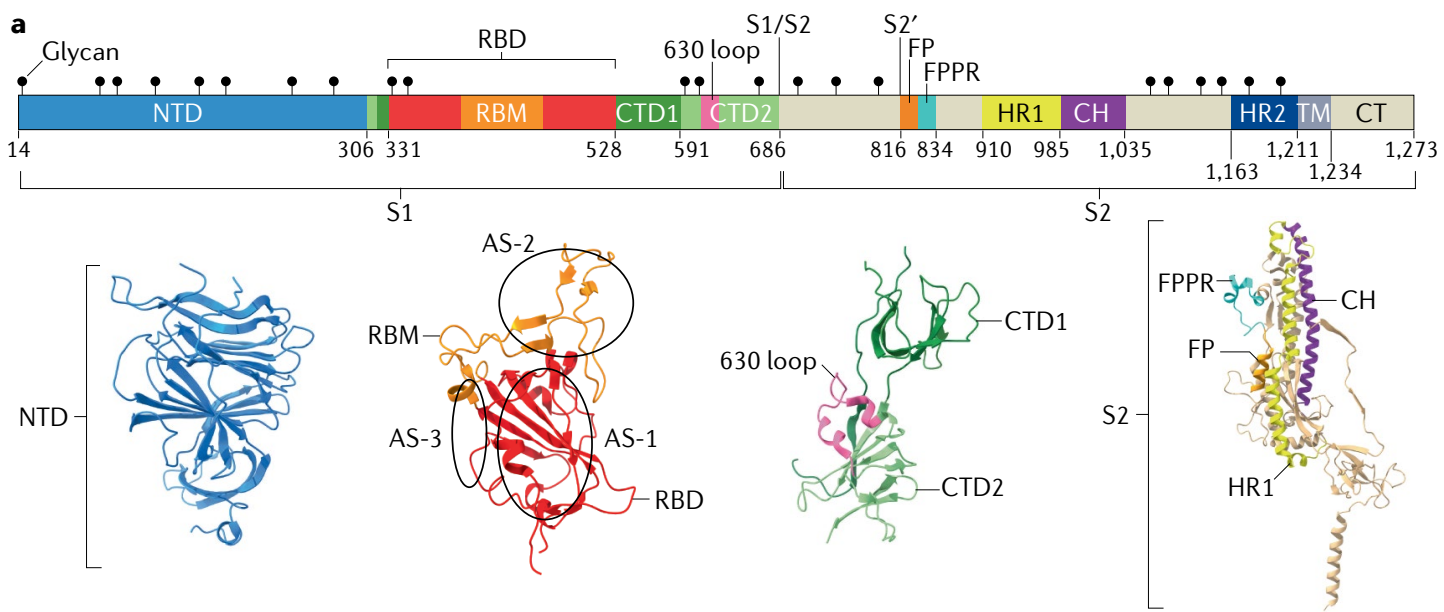

Pseudoviruses

Laboratory tools used to study and compare viral entry

processes and their inhibition. Pseudovirus particles are formed by the structural proteins of safe, non-replicative forms of viruses coated (pseudotyped) with the entry glycoprotein of a heterologous virus.

Virus-like particles Molecules similar to pseudoviruses in that they are replication incompetent but that, unlike psuedoviruses, are composed of structural proteins of the virus under study. They thus have properties (size, shape and budding site) more similar to those of the viruses from which the structural proteins are derived and can be used to study viral assembly.

Type I membrane protein A membrane protein that contains a single

transmembrane domain oriented so that its amino terminus is on the extracellular lumenal side. Synthesis of these proteins is initiated with a signal peptide that is removed in the endoplasmic reticulum.
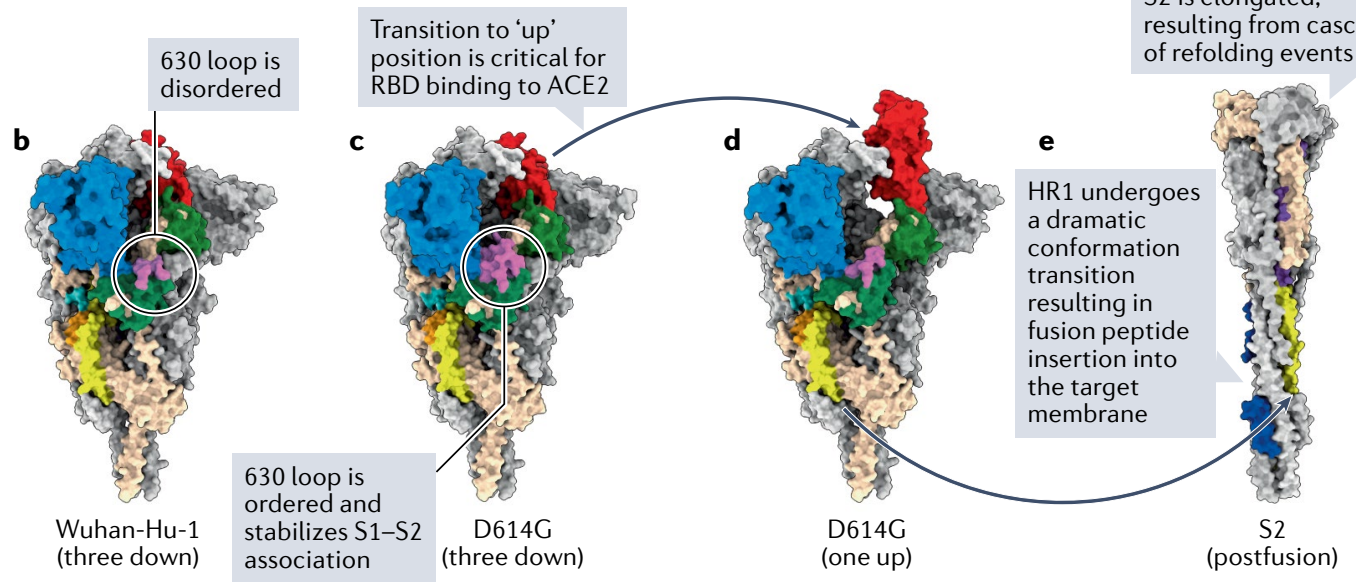

f

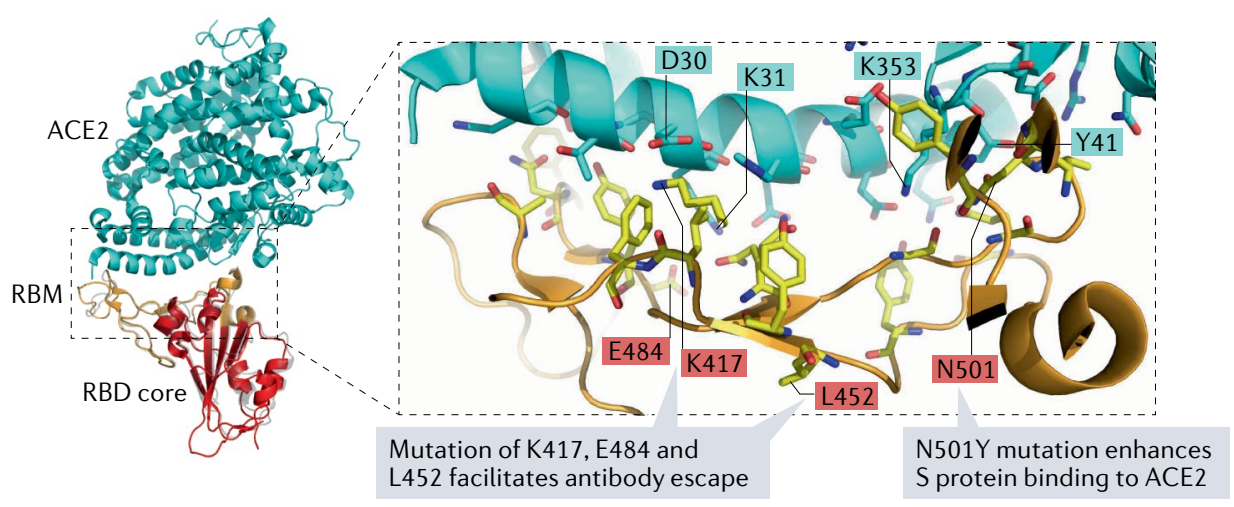

Fig. 2 | Structures of the $\mathrm{S}$ protein, its subdomains and interaction between the RBD and ACE2. a | The full-length SARS-CoV-2 spike (S) protein. Selected subdomain structures are shown in ribbon diagrams below the schematic. Three distinct receptor-binding domain (RBD) antigenic sites (AS-1, AS-2 and AS-3) ${ }^{25}$ are indicated in the RBD ribbon diagram. b-e $\mid$ Cryoelectron microscopy structures of detergent-solubilized full-length $S$ trimers from the Wuhan-Hu-1 reference strain in the conformation with three RBDs down (Protein Data Bank (PDB) ID 6XR8; part b), the D614G variant in the conformation with three RBDs down (PDB ID 7KRQ; part c), the D614G variant in the conformation with one RBD up (PDB ID 7KRR; part d) and the postfusion structure of the Wuhan-Hu-1 S2 (PDB ID 6XRA; part e). A dramatic conformational change in heptad repeat 1 (HR1) propels insertion of the fusion peptide into the target membrane. In one protomer, in the foreground, subdomains are coloured according to part $\mathbf{a}$, and the other two protomers, in the background, are shown in grey or light grey. $\mathbf{f} \mid$ The interface between angiotensin-converting enzyme 2 (ACE2; cyan) and bound RBD (red) in the ribbon diagram (PDBID 6M0J). The receptorbinding motif (RBM) is shown in orange. In the inset, 20 residues of ACE2 and 17 residues from the RBD forming networks of hydrophilic side chain interactions are shown in the stick model. Residues in the RBD that are mutated in the variants of concerns (TABLE 1) and their interacting residues in ACE2 are highlighted. CH, central helix; CT, cytoplasmic tail; CTD1, carboxyterminal domain 1; CTD2, carboxy-terminal domain 2; FP, fusion peptide; FPPR, fusion-peptide proximal region; HR2, heptad repeat 2; NTD, amino-terminal domain; S1/S2, furin-cleavage site; S2', S2' cleavage site; TM, transmembrane anchor. 
Table 1 | Spike protein mutations in SARS-CoV-2 variants

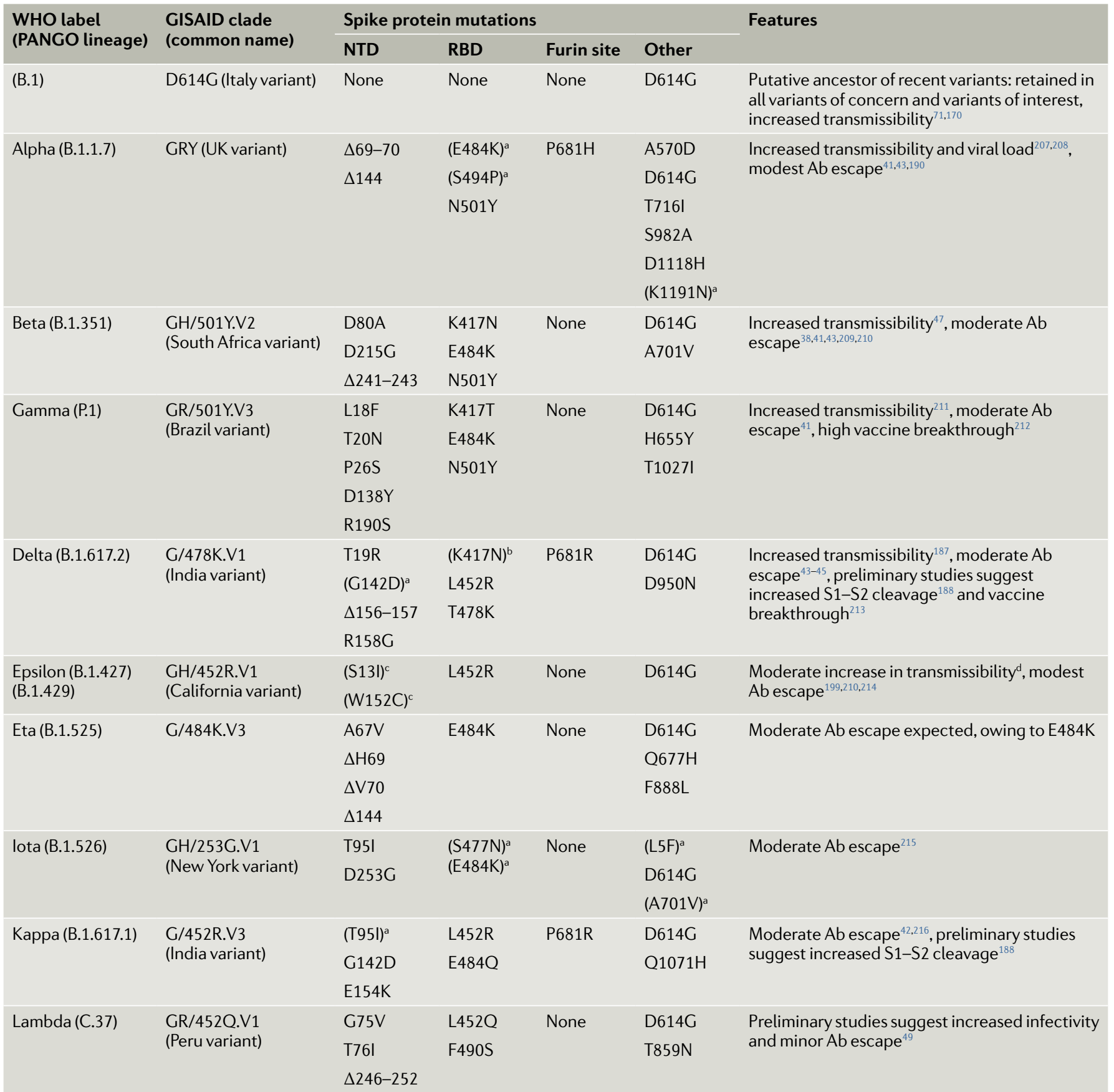

$\Delta$ indicates deletion. Ab, antibody; GISAID, Global Initiative on Sharing All Influenza Data; NTD, amino-terminal domain; PANGO, Phylogenetic Assignment of Named Global Outbreak; RBD, receptor-binding domain; WHO, World Health Organization. ${ }^{a}$ Detected in some but not all sequences. ${ }^{\mathrm{b} P r e s e n t}$ in B.1.617.2.1, Delta plus sublineage. 'Present only in B.1.429 lineage. ${ }^{\mathrm{d}} \mathrm{https} / / \mathrm{gvn} .0 \mathrm{rg} / \mathrm{covid}-19 / \mathrm{epsilon-b-1-427-b-1-429/.}$

detergent-solubilized, full-length S proteins ${ }^{25-27}$ (FIG. 2b-d), as well as those of the membrane-bound, intact $S$ proteins on the surface of the virion, which were obtained by cryo-electron tomography using chemically inactivated SARS-CoV-2 preparations of both the WuhanHu-1 strain and a B.1 variant carrying the D614G mutation $^{28-31}$ (one of the first known mutations of the $S$ protein, which was shown to prevail in other variants) (TABLE 1). These independently obtained structures are largely in agreement with each other, revealing both the overall architecture and atomic details of the protein.
In the prefusion conformation, the $\mathrm{S} 1$ subunit folds into four domains - the amino-terminal (N-terminal) domain (NTD), the RBD and two carboxy-terminal (C-terminal) domains (CTD1 and CTD2) - and wraps around the prefusion S2 subunit, which forms a central helical bundle with heptad repeat 1 (HR1) bending back towards the viral membrane (FIG. 2a). The three RBDs of the $\mathrm{S}$ trimer form the apex of the $\mathrm{S}$ protein, which samples two distinct conformations: 'up' for a receptoraccessible state and 'down' for a receptor-inaccessible state (FIG. $2 b-d$ ). In the postfusion state, conformational 
Sarbecoviruses

Coronaviruses that are related to severe acute respiratory syndrome coronavirus and belong to the subgenus Sarbecovirus in the genus Betacoronavirus. They are enveloped, positive-sense, single-stranded RNA viruses, usually use angiotensinconverting enzyme 2 as the entry receptor and infect humans, bats and other mammals. changes lead to S1 subunit disengagement from S2, and likely its dissociation from S2, while S2 undergoes a cascade of refolding events to form a stable and elongated trimer (FIG. 2e).

The NTD. The NTD is formed mainly by four stacked $\beta$-sheets and number of connecting flexible loops, bearing several $N$-linked glycans (FIG. 2a). Whether the NTD plays any functional role in SARS-CoV-2 entry remains unknown. Similar NTDs appear to facilitate the binding of sugar moieties acting as attachment factors by $\mathrm{HCoV}-\mathrm{OC} 43$ or bovine coronavirus, or the binding to the protein receptor by murine hepatitis virus $^{32,33}$. The NTD of transmissible gastroenteritis virus and MERS-CoV may facilitate prefusion-to-postfusion transition of the $S$ protein ${ }^{34,35}$. Notably, the NTD of SARS-CoV-2 is targeted by some potent neutralizing antibodies (exemplified by $4 \mathrm{~A} 8$ and $4-8$ ) $^{36,37}$, suggesting that it may be functionally important or at least located in the vicinity of other functionally critical regions, such as the RBD. Interestingly, the newly emerged variants often have mutations and deletions in the NTD, rendering them resistant to these neutralizing antibodies $^{38-49}$.

The RBD. The RBD has two subdomains (FIG. 2a): a core structure formed by a five-stranded antiparallel $\beta$-sheet covered with short connecting $\alpha$-helices on both sides, and an extended loop, named the 'receptor-binding motif' (RBM), which wraps around one edge of the core structure and makes all the contacts with ACE2 $\left(\mathrm{REFS}^{2,5,50}\right)$.
In the down conformation of the prefusion $S$ trimer, a single RBD packs against the central helical bundle of S2 and the two other RBDs, while leaning on CTD1 from the same protomer and the NTD from a neighbouring protomer (FIG. 2b,c). This configuration partially occludes the RBM, making it inaccessible to the receptor ACE2. When the RBD flips to the up conformation and fully exposes the RBM (FIG. 2d), the adjacent CTD1 and NTD also shift away to accommodate the RBD movement. Thus, the transition of the RBD from the down conformation to the up conformation is a critical step before the receptor can fully engage, and the one-RBD-up conformation appears to be a stable intermediate state. RBDs from other sarbecoviruses can be categorized into two distinct groups: human ACE2 (hACE2) binders and non-hACE2 binders ${ }^{51}$. They are likely to fold similarly because of the high degree of sequence identity in the core subdomain, but variations at the ACE2-binding interface in the RBM of the nonhACE2 binders can explain their inability to use hACE2 as an entry receptor ${ }^{51}$.

Importantly, the RBD is also the primary target of the neutralizing antibodies elicited by natural infection or vaccination (BOX 1). There are three major non-overlapping antigenic sites on the $\mathrm{RBD}^{52}$ (FIG. 2a). Most RBD-directed neutralizing antibodies, including REGN10933 (casirivimab) and C144, recognize the tip region of the RBM, and they often show remarkable potency blocking ACE2 engagement by direct competition $^{53,54}$. Another site lies on the exposed surface of the RBD when it is in the down configuration, and is

\section{Box 1 | Antibodies and antibody-like entry inhibitors for SARS-CoV-2}

Of the various stages of the virus life cycle, the entry step is one of the most attractive therapeutic targets, not only because it initiates infection but also because the cellular receptors and viral entry proteins are accessible from the extracellular space. Currently, however, the only FDA-approved antiviral drug for COVID-19, remdesivir, acts on the viral RNA-dependent RNA polymerase inside the cell rather than at the point of entry ${ }^{233}$. Camostat mesylate, a transmembrane protease, serine 2 (TMPRSS2) inhibitor, is currently under evaluation ${ }^{234}$. A multitude of other small molecules were found to inhibit SARS-CoV-2 entry in vitro via various mechanisms ${ }^{24,235-239}$, but it is premature to predict their utility because of toxicity and/or uncertain in vivo efficacy. Currently, the use of antibodies and antibody-like products is the most promising strategy to therapeutically target the entry of SARS-CoV-2.

\section{Immune plasma}

The earliest available therapy for COVID-19 was infusion of convalescent plasma. Studies show that convalescent plasma may offer modest protection against progression to severe disease or death when administered early in the course of infection ${ }^{240,241}$, but most trials in hospitalized patients showed no significant benefit ${ }^{242-244}$. Moreover, plasma therapy is complicated by the wide variation in neutralizing activity of donor plasma ${ }^{245}$.

\section{Antibodies targeting the spike protein}

Monoclonal antibodies and antibody derivatives targeting SARS-CoV-2 have been intensely studied in vitro ${ }^{246-249}$, and have been brought to market for clinical use by Regeneron and Eli Lilly. One serious problem associated with these antibodies is fast-emerging resistant mutations ${ }^{194}$. For this reason, Eli Lilly's bamlanivimab, initially authorized by the FDA as monotherapy ${ }^{250}$, is now available only as a combination with another antibody with a non-overlapping epitope, etesevimab ${ }^{251}$. Regeneron's casirivimab and imdevimab were authorized as a combination therapy, and thus far no report has indicated reduced neutralization towards recently emerged immune-escape variants. Propagation of SARS-CoV-2 in vitro or in hamsters in the presence of this antibody combination also did not elicit escape variants, although additional studies are necessary owing to the short duration of the study ${ }^{196}$. Neither cocktail is authorized for use in severely ill or hospitalized patients owing to the unfavourable risk-benefit profile in this population ${ }^{252}$.

\section{Antibodies targeting ACE2}

In contrast to anti-spike protein antibodies, antibodies targeting human angiotensin-converting enzyme 2 (ACE2) may be advantageous in that viral escape cannot arise because their target epitope is on the host cell ${ }^{253}$. Such antibodies are not expected to interfere with the function of ACE2, because the SARS-CoV-2 binding region on ACE2 does not overlap with the enzyme's active site. However, as antibodies are naturally bivalent, they likely crosslink and downregulate ACE2, which can disrupt the renin-angiotensin-aldosterone system.

\section{ACE2 mimetics}

ACE2 mimetics, such as ACE2 ectodomain, its Fc-fusion form and their variants selected for higher affinity, can prevent the initial steps of receptor engagement by the virus ${ }^{69,254-257}$. Like ACE2-targeting antibodies, viruses cannot evade them without sacrificing their ability to bind the receptor. On the one hand, catalytically inactive forms of ACE2 mimetics, in which residues involved in binding angiotensin I and angiotensin II are mutated, can be used as therapeutics without perturbing the renin-angiotensinaldosterone system ${ }^{254}$. One such construct showed enhanced rather than reduced activity against variants of concern ${ }^{257}$. On the other hand, catalytically active ACE2 mimetics may be more beneficial in treating acute respiratory distress syndrome induced by SARS-CoV-2-mediated downregulation of cellular ACE2 (REFS ${ }^{258,259}$ ). 
targeted by antibodies such as REGN10987 (imdevimab) and S309 $\left(\mathrm{REFS}^{53,55}\right)$. The third site, often referred to as a 'cryptic supersite', targeted by CR3022 (REF. ${ }^{56}$ ), is on the buried side of the RBD and is fully accessible only when the domain is in the up conformation. Together, these sites comprise the epitopes of majority of neutralizing antibodies to SARS-CoV-2, as discussed later.

The C-terminal domains. C-terminal domains are formed primarily by $\beta$-structures. The RBD appears to be an insertion between two antiparallel $\beta$-strands in CTD1, and CTD1 can also be viewed as an insertion between two antiparallel $\beta$-strands in CTD2 (FIG. 2a). Thus, a continuous strand (residues 306-330) runs through both CTD1 and CTD2, connecting the NTD and the RBD on its two ends. CTD1, packed underneath the $\mathrm{RBD}$, needs to rotate outwards with the RBD in the flipping-up transition into the receptor-accessible state. A structural element in S2, named the 'fusion-peptide proximal region' (FPPR), abutting the opposite side of CTD1 from the RBD, appears to help clamp down the RBD and stabilize the closed conformation of the $S$ trimer ${ }^{25,27}$. Therefore, CTD1 appears to be a structural relay between the RBD and the FPPR: it senses the changes in the RBD and the FPPR, which are located on either side of CTD1.

CTD2 is formed by two stacked $\beta$-sheets, each containing four strands, with a fifth strand in one sheet contributed by the connecting strand between the NTD and the RBD (FIG. 2a). In the other sheet, an interstrand loop contains the furin-cleavage site at the $\mathrm{S} 1-\mathrm{S} 2$ boundary, and one strand is the $\mathrm{N}$-terminal segment of S2. A structural element, designated the

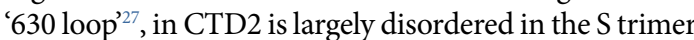
of the Wuhan-Hu-1 strain (FIG. 2b) but is ordered in the presence of the D614G mutation (FIG. 2c) and plays an important role in stabilizing the $\mathrm{S}$ trimer, as discussed later. Thus, CTD2 is another key component for the structural rearrangements of the $\mathrm{S}$ protein required for membrane fusion.

The S2 subunit. In the prefusion conformation (FIG. 2a), the S2 subunit adopts a conformation with most of the polypeptide chain packed around a three-stranded coiled coil formed by the central helices ${ }^{4,21,25}$. In particular, the coiled coil and part of HR1 together with another helix formed by residues 758-784 assemble into a ninehelix bundle, which likely contributes to overall stability of the S protein. The second half of HR1 and the so-called connector domain, which links the central helix and C-terminal heptad repeat 2 (HR2) ${ }^{57}$, surround the bottom of the coiled coil, partially protecting the fusion peptide. The FPPR, directly connected to the fusion peptide, tucks underneath CTD1 from the neighbouring protomer and also makes contacts with CTD2 of the same protomer (FIG. $2 \mathrm{~b}-\mathrm{d}$ ). The C-terminal segments, including HR2, the transmembrane domain and the cytoplasmic tail, are largely disordered in the prefusion S trimer structures, except for some low-resolution density in certain reconstructions $\mathrm{s}^{27,28}$.

In the postfusion S2 structure (FIG. 2e), HR1 and the central helix form an unusually long central three-stranded coiled coil $(\sim 180 \AA)^{25}$, almost identical to the S2 structures of SARS-CoV and mouse hepatitis virus ${ }^{57,58}$ The $\mathrm{N}$-terminal region of HR2 adopts a one-turn helical conformation and packs against the groove of the HR1 coiled coil; the C-terminal region of HR2 forms a longer helix that makes up a six-helix bundle structure with the rest of the HR1 coiled coil, forming a stable rigid postfusion structure. The postfusion S2 unexpectedly aligns $N$-linked glycans along the long axis, with four of them spaced regularly on the same side of the trimer ${ }^{25}$. Comparison of the prefusion and postfusion states of S2 suggests that HR1 undergoes a dramatic refolding transition that can insert the fusion peptide into the target cell membrane, similar to a mechanism proposed for other coronaviruses ${ }^{57,58}$.

\section{SARS-CoV-2 receptor ACE2}

ACE2 is an 805-amino acid carboxypeptidase that removes a single amino acid from the $\mathrm{C}$ terminus of its substrates. It consists of a single metallopeptidase domain in the first half that contains the HEXXH zinc-binding motif at the catalytic site and shares homology with angiotensin-converting enzyme (ACE), and a C-terminal half including the transmembrane domain that shares homology with collectrin ${ }^{59,60}$. Integral to the renin-angiotensin-aldosterone system, the primary role of ACE2 in normal physiology is to convert angiotensin I and angiotensin II, generated by renin and ACE, into angiotensin-(1-9) and angiotensin-(1-7), respectively ${ }^{60-62}$. Like ACE2 for SARS-CoV-2, the obligate receptors for several other coronaviruses are also proteases (DPP4 (also known as CD26) for MERS-CoV and $\mathrm{APN}$ for $\mathrm{HCoV}-229 \mathrm{E})^{63-65}$. Interestingly, the catalytic activity of these receptors neither is necessary for their receptor function nor overlaps with the virus-binding site. Consistently, small-molecule inhibitors of the catalytic site of ACE2 do not block infection by SARS-CoV ${ }^{66}$. Nonetheless, studies have shown that downregulation of ACE2 resulting from SARS-CoV infection contributes to disease severity by perturbing the renin-angiotensin-aldosterone system $^{67}$. The same is presumed for SARS-CoV-2, given that SARS-CoV and SARS-CoV-2 engage ACE2 in the same manner.

Interaction between the $S$ protein and the receptor. The interface between the RBD of the S protein and ACE2 is formed primarily by a gently concave outer surface of the extended RBM and the N-terminal helix of the receptor ${ }^{2,5}$ (FIG. 2f). There are 20 residues of ACE2 and 17 residues from the RBD forming networks of hydrophilic, side chain interactions. Of the latter, residues Lys417, Leu452, Glu484 and Asn501 are those that have been mutated in the newly emerged SARS-CoV-2 variants of concern ${ }^{44-49}$ (TABLE 1), and may confer both increased receptor binding and/or antibody resistance. As expected, the mode of ACE2 binding is almost identical between the SARS$\mathrm{CoV}$ and SARS-CoV-2 RBDs ${ }^{2,5,50}$. Many interacting residues are identical or have only conservative substitutions between the two RBDs. Several positions with non-conservative substitutions can still maintain similar interactions. The only extra ACE2-interacting residue in 
SARS-CoV-2 is Lys417, which forms a salt bridge with Asp30 of ACE2 (FIG. 2f). Interestingly, despite the higher affinity of the SARS-CoV-2 RBD for ACE2 compared with the SARS-CoV RBD, the SARS-CoV-2 S protein trimer does not bind ACE2 as efficiently as does the SARS-CoV S protein trimer ${ }^{10,21}$. This apparent paradox, a consequence of the inherent instability of the original SARS-CoV-2 (Wuhan-Hu-1) S protein, is further discussed together with the D614G mutation later. Other shared features of the RBD-ACE2 interfaces between the two viruses include the role of multiple tyrosine residues forming hydrogen bonds at the interface and a common disulfide-bonded RBD core ${ }^{2,5,50}$.

The structures of the soluble SARS-CoV-2 S trimer in complex with monomeric ACE2 confirm that the receptor interacts with the RBD in its up conformation ${ }^{68,69}$, which is consistent with previous findings with ACE2 binding to the SARS-CoV S protein ${ }^{70}$. While the NTD shifts outwards slightly, the S2 subunit remains largely unchanged upon ACE2 binding.

ACE2 expression and the putative impact of comorbidities. Analysis of animal models and human transcriptome databases suggests that ACE2 expression in the lower lung is relatively limited to type II alveolar cells, but is higher in the upper bronchial epithelia and much higher in the nasal epithelium, especially in the ciliated cells ${ }^{71-76}$. This difference in ACE2 expression level in the respiratory tract is mirrored by the SARSCoV-2 infection gradient, with nasal ciliated cells being primary targets for SARS-CoV-2 replication in the early stage of infection ${ }^{71,75}$. Despite the respiratory route being dominant in SARS-CoV-2 infection, the highest levels of ACE2 expression are found in the small intestine, testis, kidney, heart muscle, colon and thyroid gland ${ }^{73,77}$. Cardiac infection by SARS-CoV-2 was frequently found in autopsy cases ${ }^{78}$, and the presence of ACE2 in colon and kidney cells has been suggested as an explanation for gastrointestinal and renal complications of SARS-CoV-2 infection. ACE2 expression in the gastrointestinal tract is consistent with the observation that many coronaviruses, including sarbecoviruses, are transmitted via the faecaloral route as well as the respiratory route. Inflammatory cytokines released in severe COVID-19, such as IL- $1 \beta$ and type I and type III interferons, can upregulate ACE2 expression, potentially establishing a positive-feedback loop for viral replication ${ }^{71,79-81}$. However, their effect on disease severity is unknown. Moreover, a recent report indicates that interferon-stimulated expression of ACE2 yields a truncated isoform that cannot support SARS-CoV-2 binding ${ }^{82}$.

Several health comorbidities, including hypertension, hyperlipidaemia, diabetes, chronic pulmonary diseases, old age and smoking, are risk factors for COVID-19. A number of these factors have been proposed to modulate ACE2 expression. Differences in ACE2 levels associated with age and sex are controversial; advanced age has been both positively ${ }^{83,84}$ and negatively ${ }^{85}$ correlated with ACE2 expression, while another independent study found no significant effect ${ }^{80}$. Male sex has similarly been associated with higher ACE2 expression in some studies ${ }^{84}$ but not others ${ }^{80,85}$. Widely accepted epidemiological data indicate that a history of smoking tobacco increases the risk of severe disease ${ }^{86,87}$. However, whether smoking causes upregulation of ACE2 and is associated with enhanced infection is unclear. Many biochemical studies have shown that ACE2 expression is increased in lung tissue samples from smokers and patients with chronic obstructive pulmonary disease $\mathrm{e}^{80,88,89}$ and also in mouse lungs exposed to cigarette smoking ${ }^{80}$. However, other studies indicated that ACE2 expression in the airway is not affected by smoking ${ }^{76}$, and that smoking is not a significant epidemiological risk factor for COVID-19 $\left(\mathrm{REFS}^{90,91}\right)$. A limited number of studies showed that diabetes mellitus is associated with increased ACE2 expression in human sputum cells and in murine kidney tissue $\mathrm{e}^{77,92}$ and that cystic fibrosis is associated with ACE2 (and TMPRSS2) upregulation in human lung specimens ${ }^{71}$. It was initially speculated that common antihypertensive drugs such as ACE inhibitors and angiotensin receptor blockers could upregulate ACE2 expression $^{93}$, raising concern that use of these agents could increase disease severity ${ }^{94,95}$. Although this idea is consistent with the association between hypertension and severer COVID-19 outcomes, recent studies have refuted this hypothesis ${ }^{76,91,96,97}$. Thus, to date, no comorbidity has been unambiguously associated with ACE2 expression level.

ACE2 orthologues in potential reservoir species. Similarities in virus-binding hotspots of ACE2 and its orthologues in reservoir animal species contribute to the zoonotic potential of sarbecoviruses. Horseshoe bats of the genus Rhinolophus are the presumed longterm reservoirs of SARS-CoV and SARS-CoV-2, but it remains unclear exactly which species in the genus serves as the most recent bat host of each virus ${ }^{98}$. A mammalian intermediate host is thought to facilitate transmission of both viruses to humans. Accordingly, SARS-CoV has been isolated from palm civets (Paguma larvata) and raccoon dogs (Nyctereutes procyonoides) ${ }^{99}$, and viruses close to SARS-CoV-2 were isolated from pangolins (Manis javanica) ${ }^{100-102}$. The significance of receptor adaptation during host-jumping from an intermediate host to humans is underscored by evidence that two independent zoonotic events gave rise to the two SARS outbreaks between 2002 and 2004 with markedly different disease severity. While the 2002-2003 SARS epidemic resulted in nearly $10 \%$ mortality, the outbreak in 2003-2004 resulted in a much milder disease ${ }^{103}$. Analysis of the S protein of the virus isolates from both outbreaks revealed that reduced disease severity of the second outbreak was in part due to insufficient adaptation of the S protein to hACE2, as shown by lower affinity $^{66}$. This affinity difference was mapped to a single residue in the RBD, foreshadowing the impact of RBD mutations in SARS-CoV-2 on transmissibility and disease severity.

Viruses closely related to SARS-CoV-2 have been isolated from the horseshoe bat, Rhinolophus affinis, including SARS-like betacoronavirus RaTG13, which shares $96 \%$ nucleotide identity with SARS-CoV-2 $\left(\mathrm{REF}^{104}\right)$. Investigators evaluated the receptor function of a wide array of bat ACE2 orthologues and found 
that only 9 of 46 supported the entry by SARS-CoV-2 pseudoviruses ${ }^{105}$. Replacement of five residues in hACE2 with residues originating from the SARS-CoV-2competent ACE2 orthologues of Rhinolophus spp. increased its binding affinity for the SARS-CoV-2 $\mathrm{RBD}^{98}$. Although not fully conclusive, these data are consistent with a Rhinolophus origin of SARS-CoV-2. Phylogenetic analysis and confirmatory infection studies have shown that ACE2 orthologues from a wide range of mammals, including domestic animals and livestock, support SARS-CoV-2 infection, suggesting that many animals have the potential to act as intermediate hosts as well ${ }^{106}$.

\section{Additional host entry factors}

In addition to ACE2, several molecules have been suggested to serve as alternative receptors for SARS-CoV and SARS-CoV-2. These include C-type lectins, DC-SIGN and L-SIGN ${ }^{107-109}$. Lectins are involved in the recognition of a broad range of pathogen $\mathbf{s}^{110}$ and mediate intercellular adhesion ${ }^{111}$. They bind a wide range of viruses by recognizing the glycans on the virion surface, often promoting viral entry by allowing the virus to attach to the target cell. Likewise, TIM1 and AXL were also suggested to be alternative SARS-CoV-2 receptors ${ }^{112,113}$. As members of phosphatidylserine receptor families, TIM and TAM, respectively, they enhance the entry of a wide range of enveloped viruses by binding to phosphatidylserine on the virion membrane $e^{114-116}$. Although lectins and phosphatidylserine receptors increase viral entry, they are non-specific and do not support efficient infection by SARS-CoV or SARS-CoV-2 in the absence of ACE2 (REFS ${ }^{115,117}$ ), and thus 'attachment factors' would better describe those molecules.

Similarly, CD147, a transmembrane glycoprotein expressed ubiquitously in epithelial and immune cells, was proposed to be an alternative receptor for SARS-CoV and SARS-CoV-2 infection ${ }^{118,119}$. Although a modest increase in viral entry was observed with higher levels of CD147, and although its upregulation was observed in obesity and diabetes ${ }^{120}$, which are potential risk factors for severe COVID-19, the role of CD147 in SARS-CoV-2 infection has been disputed on the basis of the inability of CD147 to bind the S protein ${ }^{121}$. Two groups identified neuropilin 1 (NRP1) as a host factor for SARS-CoV-2 (REFS ${ }^{122,123}$ ). Although NRP1 is expressed in olfactory and respiratory epithelial cells ${ }^{122}$, its expression is low in ciliated cells, the primary target cells for SARS-CoV-2 in the airway, while it is high in goblet cells, which are not susceptible to SARS-CoV-2 $\left(R_{E F S}{ }^{11,75}\right)$. Nonetheless, NRP1 was shown to enhance TMPRSS2-mediated entry (see the next section) of wild-type SARS-CoV-2 but not that of mutant virus that lacks the multibasic furin-cleavage site ${ }^{122}$. NRP1 was also shown to bind $\mathrm{S} 1$ through the multibasic furin-cleavage site and to promote $\mathrm{S} 1$ shedding and to expose the S2' site to TMPRSS2 (REF. ${ }^{124}$ ). Recently, the structure of ACE2 in complex with a neutral amino acid transporter, B0AT1, was analysed by cryo-EM in the presence and absence of the SARS-CoV-2 S protein ${ }^{23}$. ACE2 was previously shown to be essential for B0AT1 expression in the small intestine ${ }^{125}$. While B0AT1 is expressed in the gastrointestinal tract and kidney, it is not present in the lung. However, a B0AT1 homologue in the lung might contribute to SARS-CoV-2 infection. Additional studies are warranted to confirm the role of NRP1 and B0AT1 in SARS-CoV-2 infection.

\section{SARS-CoV-2 entry process}

Viral entry proteins must fold into an energetically stable state, and yet must undergo a subsequent conformational transition that provides sufficient energy to overcome the natural repulsion between the virus and the cellular membranes. Therefore, the S protein transitions to a so-called metastable state, a state prone to transformation to a lower-energy state, before membrane fusion. Like in SARS-CoV and other coronaviruses, this $S$ protein transition is enabled through two proteolytic cleavage steps following ACE2 engagement. The first of these is localized to the S1-S2 boundary, and the second is localized to the S2' site in the $\mathrm{S} 2$ subunit. For SARS-CoV, both sites are cleaved by proteases in the target cell. In the case of SARS-CoV-2, the $\mathrm{S} 1-\mathrm{S} 2$ boundary is cleaved by furin in the virusproducer cell, whereas the $\mathrm{S} 2$ ' site cleavage still requires target-cell proteases. Cell entry by both viruses is therefore dependent on the target-cell proteases, and TMPRSS2 and cathepsin L are the two major proteases involved in S protein activation. As TMPRSS2 is present at the cell surface, TMPRSS2-mediated $S$ protein activation occurs at the plasma membrane, whereas cathepsin-mediated activation occurs in the endolysosome (FIG. 3).

Cleavage of the S protein S1-S2 boundary by furin. The presence of a multibasic site (Arg-Arg-Ala-Arg) located at the S1-S2 junction, which is cleaved by furin (FIG. 1), distinguishes SARS-CoV-2 from SARS-CoV and all other known sarbecoviruses whose $S$ protein is not cleaved by furin-like proteases during virus maturation in the infected cell. Cleavage of the S1-S2 boundary is a prerequisite for the cleavage of the $S 2^{\prime}$ site $^{126}$, and both cleavage events are essential to initiate the membranefusion process. The entry glycoproteins of many viruses, including HIV-1 and avian influenza viruses, are cleaved by furin, but such cleavage does not destabilize the entry glycoprotein; the two subunits are associated via a stable interaction. By contrast, the S1 subunit of human SARS-CoV-2 Wuhan-Hu-1 strain is easily shed from the S2 subunit and thereupon assumes a premature postfusion conformation of the S2 trimer, a non-functional form of the $\mathrm{S}$ protein (FIG. 2e). This perplexing observation suggests that the acquisition of a furin-cleavage site by SARS-CoV-2 may have been a recent event. While it would have been relatively straightforward for the virus to have eliminated this furin-cleavage site and depend on the target-cell proteases as does SARS-CoV, SARS$\mathrm{CoV}-2$ rather acquired a different mutation, D614G, to stabilize the S protein and slow S1 shedding ${ }^{27,127}$. This steadfast retention of the destabilizing furin site indicates that this site is important for virus fitness in human hosts. Indeed, the furin site was recently shown to be an important determinant for SARS-CoV-2 transmission among co-housed ferrets ${ }^{128}$. 


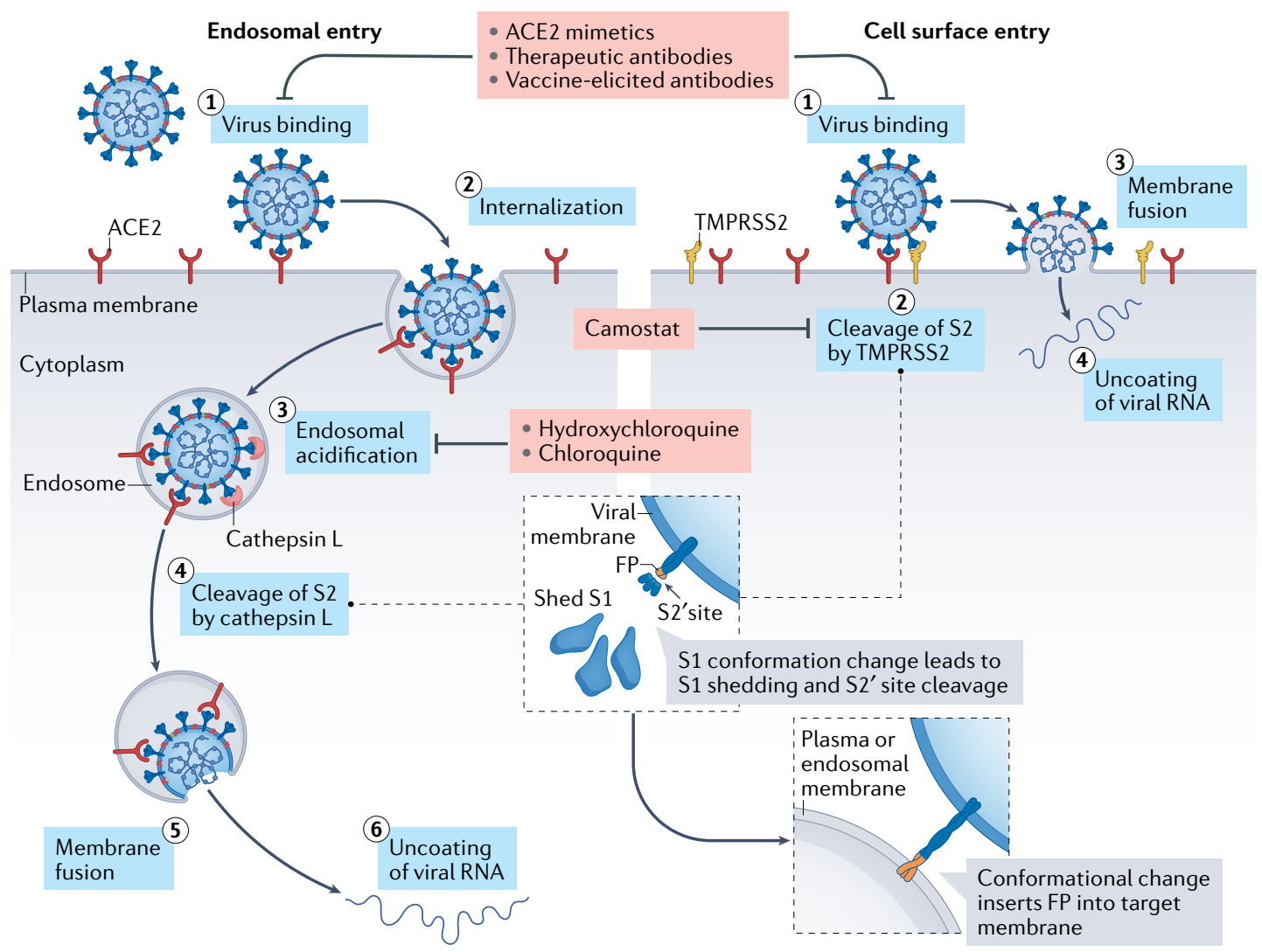

Fig. 3 | Two distinct SARS-CoV-2 entry pathways. Two spike (S) protein cleavage events are typically necessary for the coronavirus entry process: one at the junction of the $\mathrm{S} 1$ and $\mathrm{S} 2$ subunits and the other at the $\mathrm{S} 2$ ' site, internal to the S2 subunit. In the case of SARS-CoV-2, the polybasic sequence at the S1-S2 boundary is cleaved during virus maturation in an infected cell, but the $\mathrm{SZ}^{\prime}$ site is cleaved at the target cell following angiotensin-converting enzyme 2 (ACE2) binding. Virus binding to ACE2 (step 1) induces conformational changes in the S1 subunit and exposes the S2' cleavage site in the S2 subunit. Depending on the entry route taken by SARS-CoV-2, the S2' site is cleaved by different proteases. Left: If the target cell expresses insufficient transmembrane protease, serine 2 (TMPRSS2) or if a virus-ACE2 complex does not encounter TMPRSS2, the virus-ACE2 complex is internalized via clathrin-mediated endocytosis (step 2) into the endolysosomes, where S2' cleavage is performed by cathepsins, which require an acidic environment for their activity (steps 3 and 4). Right: In the presence of TMPRSS2, S2' cleavage occurs at the cell surface (step 2). In both entry pathways, cleavage of the S2' site exposes the fusion peptide (FP) and dissociation of S1 from S2 induces dramatic conformational changes in the S2 subunit, especially in heptad repeat 1, propelling the fusion peptide forward into the target membrane, initiating membrane fusion (step 5 on the left and step 3 on the right). Fusion between viral and cellular membranes forms a fusion pore through which viral RNA is released into the host cell cytoplasm for uncoating and replication (step 6 on the left and step 4 on the right). Several agents disrupt interaction between the S protein and ACE2: ACE2 mimetics, therapeutic monoclonal antibodies targeting the neutralizing epitopes on the $S$ protein and antibodies elicited by vaccination block virus binding to ACE2 and thus inhibit both entry pathways. By contrast, strategies targeting post-receptor-binding steps differ between the two pathways. Being a serine protease inhibitor, camostat mesylate restricts the TMPRSS2-mediated entry pathway. Hydroxychloroquine and chloroquine block endosomal acidification, which is necessary for cathepsin activity, and thus restrict the cathepsin-mediated entry pathway.

Type II transmembrane protein

A membrane protein that contains a single

transmembrane segment with its amino terminus on the cytoplasmic side and its carboxy terminus on the extracellular/lumenal side of the membrane.
Role of TMPRSS2 in viral entry. After the S1-S2 boundary is cleaved, the $\mathrm{S} 2$ ' site must also be cleaved to fully activate the fusion process either by TMPRSS2 on the cell surface or by cathepsins in the endosomes (FIG. 3). TMPRSS2 is a type II transmembrane protein with serine protease activity whose major physiological role and substrate specificity are not well defined. Nonetheless, its role in respiratory virus infection, especially for influenza viruses ${ }^{129-131}$ and SARS coronaviruses ${ }^{12-14}$, is well established. TMPRSS2 is found in the gastrointestinal, respiratory and urogenital epithelium ${ }^{132,133}$. Among these tissues, three major cell types co-express TMPRSS2 and ACE2 - type II pneumocytes, ileal absorptive enterocytes and nasal goblet secretory cells ${ }^{81}$ - although other studies showed that nasal ciliated cells but not nasal goblet cells express ACE2 (REFS ${ }^{71,76}$ ) or that both cells express ACE2 at high levels ${ }^{72}$. ACE2 expression in the lower airway is limited, but ACE2 is expressed at a higher level in the upper airway, and indeed many airway cells that express ACE2 also express TMPRSS2 $\left(\mathrm{REFS}^{74,134-136}\right.$ ). While TMPRSS2 is the most frequently studied protease in coronavirus entry, other serine proteases present in the lung, including human airway trypsin-like protease (HAT), TMPRSS4, TMPRSS11A, TMPRSS11E and matriptase, as well as secreted neutrophil elastase, appear to contribute to infection by many 
respiratory viruses ${ }^{137}$. Studies on their involvement in viral infection in vivo will provide further insight into the SARS-CoV-2 entry pathway and the means to inhibit the process.

Although both viruses utilize TMPRSS2, SARS-CoV is less dependent on TMPRSS2 than is SARS-CoV-2 $\left(\mathrm{REFS}^{138,139}\right)$. One determining factor might be the presence or absence of a furin site at the S1-S2 boundary ${ }^{140}$. It is possible that the sequence of the $\mathrm{S} 1-\mathrm{S} 2$ junction of the SARS-CoV S protein is not a suitable substrate for TMPRSS2 but is more easily cleaved by cathepsins. This explanation is consistent with the observation that replacing the SARS-CoV-2 furin site with the equivalent sequence of SARS-CoV or RaTG13 virus from horseshoe bats, containing no multibasic site, prevented efficient SARS-CoV-2 infection of TMPRSS2 ${ }^{+}$human airway cells ${ }^{9}$.

Role of cathepsins in viral entry. Although SARS-CoV-2 prefers activation by TMPRSS2, cleavage of the S2' site can also be mediated by cathepsins, especially cathepsin L (FIG. 3). If the target cells express insufficient TMPRSS2 or if a virus-ACE2 complex does not encounter TMPRSS2, ACE2-bound virus is internalized via clathrin-mediated endocytosis ${ }^{17,18}$ into the late endolysosome, where the S2' site is cleaved by cathepsins. Presumably owing to multiple ligations to ACE2, binding of SARS-CoV, SARS-CoV-2 or purified $S$ protein induces ACE2 endocytosis ${ }^{17,18}$. The role of cathepsins in processing the S2' site is supported by partial inhibition of pseudovirus entry by cathepsin L inhibitors in TMPRSS2 $2^{+}$cells $^{138,141}$.

Cathepsins are non-specific proteases with endopeptidase and exopeptidase activities that participate in protein degradation in the late endosomes and lysosomes. They are divided into three catalytic classes: aspartic (D and $E$ ), serine $(G)$ and cysteine (B, C, K, L, $\mathrm{S}$ and $\mathrm{V}$ ) proteases. Of these, cysteine proteases (cathepsins B, L and S) contribute the most to viral entry. The role of cathepsins in viral entry is derived mostly from studies on reoviruses, Ebola virus and SARS-CoV ${ }^{142-144}$, with limited reports on SARS-CoV-2 (REF. $\left.{ }^{138}\right)$. Whereas cathepsin B plays an essential role in Ebola virus entry, cathepsin L plays a greater role in SARS-CoV ${ }^{15,16,142}$ and SARS-CoV-2 (REFS ${ }^{138,141}$ ) entry. The lower dependence of SARS-CoV-2 on the endosomal pathway explains the limited effect of endosomal acidification inhibitors such as hydroxychloroquine on restricting SARS-CoV-2 infection of target cells ${ }^{138}$. In one study, while the potency of hydroxychloroquine was dramatically impaired when the target cell expressed TMPRSS2, it was partially restored in the presence of a TMPRSS2 inhibitor ${ }^{138}$, suggesting a potential benefit of combined use of a TMPRSS2 inhibitor and hydroxychloroquine.

Membrane fusion. Recent structural studies identified key components of the $S$ fusion machinery, including the FPPR, 630 loop and CTD2, which appear to modulate the fusogenic structural rearrangements of the $S$ protein (FIG. 2). The FPPR and the 630 loop help maintain the RBDs in the down conformation but move out of their positions when the adjacent RBD flips up.
As summarized in FIG. 4, the RBD could sample the up conformation due to intrinsic protein dynamics. If ACE2 captures the RBD-up conformation, expelling both the 630 loop and the FPPR from their positions in the closed $S$ trimer conformation, the FPPR shift may help expose the S2' site near the fusion peptide for proteolytic cleavage. Departure of the 630 loop from the hydrophobic surface of CTD2 can destabilize this domain and free the $\mathrm{N}$-terminal segment of S2 from S1, likely releasing S1 altogether, owing to the precleavage of the S1-S2 boundary of the SARS-CoV-2 $S$ protein by furin as discussed earlier. Dissociation of S1 would then initiate a cascade of refolding events in the metastable prefusion S2, allowing the fusogenic transition to a stable postfusion structure (FIG. 4). Accompanying these transitions, the thrust of HR1 unfolding drives fusion peptide insertion into the target-cell membrane ${ }^{57,58}$. Folding back of HR2 places the fusion peptide and transmembrane segments at the same end of the molecule; this proximity causes the membranes with which they interact to bend towards each other, effectively leading to membrane fusion. This model is also very similar to that proposed for membrane fusion catalysed by the HIV envelope protein, in which gp120 dissociation triggers refolding of gp41 to complete the fusion process ${ }^{145}$.

Cellular proteins restricting viral entry. Toll-like receptors (TLRs) recognize pathogen-associated molecular patterns and induce the production of type I interferons. Of these, TLR3, TLR7, TLR8 and TLR9 mount antiviral immune responses: TLR3 recognizes double-stranded RNA viruses, TLR9 recognizes unmethylated CpG in viral DNA, and, relevant to coronaviruses, TLR7 and TLR8 bind G/U-rich single-stranded viral RNA. TLR7 and TLR8 reside in the endosomes and were shown to induce proinflammatory cytokines in response to SARS-CoV and SARS-CoV-2 RNA ${ }^{146,147}$. TLR7 and TLR8 are both expressed in lung tissue. Although TLR7 is expressed at higher levels in the brain, skin and lymphoid tissues than in the lung, TLR8 is predominantly expressed in the lung and lymphoid tissues.

Many interferon-stimulated gene products were identified as important for SARS-CoV-2 replication, but only a few of them are involved in the entry steps: interferon-induced transmembrane proteins (IFITMs) ${ }^{148,149}$ and lymphocyte antigen 6 family member $E$ (LY6E) ${ }^{150,151}$. Four members of the human IFITM family (IFITM1, IFITM2, IFITM3 and IFITM5) are constitutively expressed at a high level but are strongly induced by type I and type II interferons and were identified as cellular antiviral proteins against influenza A viruses and flaviviruses ${ }^{152}$ and later against filoviruses and SARS-CoV ${ }^{153}$. Recently, IFITM2 was shown to restrict SARS-CoV-2 entry ${ }^{149}$. IFITM proteins prevent viruses from traversing the endosomal membrane to access cellular cytoplasm by an unclear mechanism. Such a restriction can be bypassed if SARS-CoV were directed to enter cells exclusively at the plasma membrane ${ }^{153}$. This restriction is amplified if the furin site is removed from SARS-CoV-2 (REF. ${ }^{149}$ ), and is compensated by TMPRSS2 overexpression $^{148,151}$. In addition, it does not affect the viruses that enter the host cell solely via fusion at the 

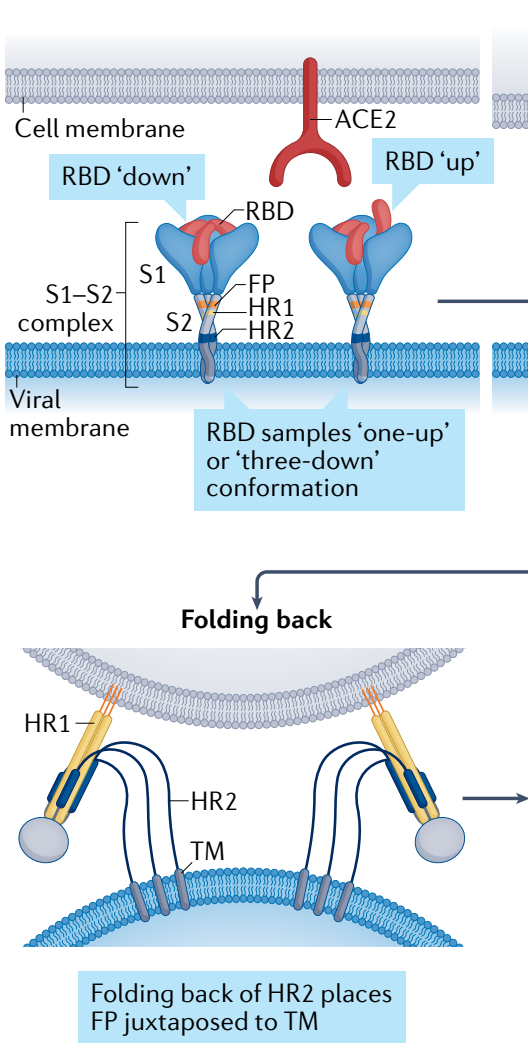

\section{Postfusion state and viral penetration}

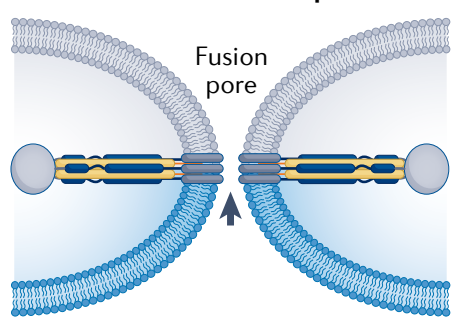

Membranes in proximity form fusion pore, allowing viral genome release into the cell

Fig. 4 | A model for membrane fusion induced by the SARS-CoV-2 $\mathrm{S}$ protein. Structural transition from the prefusion conformation to the postfusion conformation inducing membrane fusion likely proceeds stepwise as follows. The prefusion spike (S) protein trimer fluctuates between the three receptor-binding domain (RBD)-down, closed conformations and one RBD-up, open conformation. RBD binding to angiotensin-converting enzyme 2 (ACE2) enables exposure of the S2' cleavage site immediately upstream of the adjacent fusion peptide (FP). Cleavage at the S2' site releases the structural constraints on the FP and initiates a cascade of refolding events in S2, probably accompanied by complete dissociation of S1. Formation of the long central three-stranded coiled coil and folding back of heptad repeat 2 (HR2) leads to the postfusion structure of S2 that brings the two membranes together, facilitating fusion pore formation and viral entry. As shown in FIG. 3, these events can occur either at the plasma membrane or in the endosomal compartment. HR1, heptad repeat 1; TM, transmembrane segment.

plasma membrane $\mathrm{e}^{153}$. These observations indicate that the site of membrane fusion is crucial for the antiviral activity of IFITM proteins.

LY6E is a glycophosphatidylinositol-anchored cell surface protein and was shown to inhibit replication of vesicular stomatitis virus and mouse hepatitis virus ${ }^{154}$, but it was also identified to promote yellow fever virus replication ${ }^{155}$, HIV-1 entry ${ }^{156}$, flavivirus internalization ${ }^{157}$ and influenza A virus entry ${ }^{158}$. Proposed mechanisms for entry enhancement include formation of a microtubulelike network that likely guides endocytosed viruses along the tubules ${ }^{157}$ and promotion of uncoating steps following membrane fusion ${ }^{158}$. Recently, LY6E was shown to impair infection by SARS-CoV, SAR-CoV-2 and MERS-CoV by inhibiting the $S$ protein-mediated membrane fusion, and mice lacking LY6E expression in immune cells were highly susceptible to mouse hepatitis virus, also a coronavirus ${ }^{150}$. Unlike IFITM-mediated restriction, LY6E-mediated inhibition was not overcome

by TMPRSS2 expression ${ }^{151}$. Further study is warranted to clarify the distinct roles of LY6E in regulating infection with SARS-CoV-2 and other viruses.

\section{Natural evolution of the $S$ protein}

Comparison of the $\mathrm{S}$ protein sequences indicates SARS-CoV-2 may have emerged from the recombination between bat and pangolin coronaviruses. During zoonosis, SARS-CoV-2 acquired a furin-cleavage site at the boundary of the S1 and S2 domains. The virus retained this cleavage site throughout the pandemic but acquired the D614G mutation to compensate for S protein instability. More recently, as the number of infected or vaccinated people increases, SARS-CoV-2 has evolved to acquire $S$ protein mutations to escape neutralizing antibodies.

\section{$S$ proteins from sarbecoviruses in reservoir species.} Specimens taken from pangolins (Manis javanica) were found to be infected with coronaviruses similar in sequence to SARS-CoV-2 and capable of utilizing ACE2 for entry ${ }^{100-102}$. However, the pangolin is not likely the long-term reservoir species for SARS-CoV-2, because most infected pangolins exhibited severe respiratory distress and died within weeks ${ }^{102}$. In addition, because pangolin coronaviruses share lower sequence homology with SARS-CoV-2 than does RaTG13, a bat isolate, it is unlikely that a particular pangolin coronavirus is directly linked to the present SARS-CoV-2 outbreak. Interestingly, whereas pangolin isolates exhibit higher homology in the RBD with SARS-CoV-2 than does RaTG13, RaTG13 shares much greater homology with SARS-CoV-2 than do pangolin isolates outside the $\mathrm{RBD}^{3,100,102}$. This raises a possibility that the pangolin coronavirus RBD was introduced into the $S$ gene of RaTG13 or another close ancestor of SARS$\mathrm{CoV}-2$ through a recombination event ${ }^{104,159}$. It remains unknown in which species, if any, this recombination event occurred. Other analyses suggest SARS-CoV-2 did not acquire the RBD from a pangolin coronavirus but rather that it evolved in bats and gained the ability to infect humans and pangolins ${ }^{160,161}$. Furthermore, other studies suggest SARS-CoV-2 crept into humans much earlier than 2019 through unnoticed infection and obtained its unique features, RBD and furin-cleavage site $^{160,162}$.

Adaptation to humans. It was suggested that the acquisition of the furin-cleavage site in the SARS-CoV-2 $S$ protein was essential for zoonotic transfer to humans ${ }^{9,163}$, and experimental data confirmed that SARS-CoV-2 pseudoviruses lacking this cleavage site in the $S$ protein are incapable of facilitating entry into human airway cells ${ }^{9}$. Notably, the furin site is not essential for infection of mammalian epithelial cells generally, as it is lost after a few passages of the virus in Vero cells (African green monkey kidney epithelial cells $)^{28,164-167}$. However, recent preliminary studies show virus passage in TMPRSS2overexpressing Vero cells or in human lung cells prevents deletion or mutation of this site ${ }^{167-169}$. Therefore, acquisition of the furin-cleavage site appears to be one of the first human adaptation events. 
For several months as SARS-CoV-2 initially spread throughout the world, only one $S$ protein mutation, D614G, exhibited clear evidence of positive selection ${ }^{170,171}$. Although acquisition of the furin site by SARS-CoV-2 and the resulting $\mathrm{S}$ protein cleavage appear to be essential for human infection, they also makes the virus less infectious than SARS-CoV by rendering the $\mathrm{S}$ protein prone to $\mathrm{S} 1$ shedding, as discussed earlier. To compensate for this disadvantage, the SARS-CoV-2 S protein appears to have gained stronger intermolecular association between the S1 and S2 subunits, by way of the D614G mutation. A number of studies have supported such a stabilizing effect as the mode of infectivity enhancement of the D614G variant virus $^{27,127,172-174}$. Cryo-EM studies of full-length $S$ trimers revealed the source of the increased $S$ protein stability: the D614G mutation renders the 630 loop more ordered, securing the NTD and CTD1 and resulting in reduced S1 shedding ${ }^{25,27}$ (FIG. 2b,c). A biochemical study clearly showed increased functional S protein density on the G614-containing variant as a result of reduced S1 shedding ${ }^{127}$. Others have explained that a greater proportion of the one-RBD-up conformation among G614-containing $\mathrm{S}$ proteins is associated with increased virus infectivity ${ }^{22,175-177}$. Most such studies, however, were performed with non-native S protein lacking the furin site and/or carrying the diproline mutation, which has been shown to stabilize the prefusion conformation ${ }^{21}$. Regardless of its mechanism for enhanced infectivity, faster transmission of D614G virus was demonstrated ${ }^{71}$. The fact that most currently circulating SARS-CoV-2 isolates carry the D614G mutation indicates it is undoubtedly a beneficial mutation for adaptation to humans.

Additional adaptations to the human host are ongoing. The SARS-CoV-2 variants Alpha (lineage B.1.1.7), Beta (B.1.351) and Gamma (P.1), which were first identified in the United Kingdom, South Africa and Brazil, respectively, carry a common N501Y mutation in addition to the D614G mutation (TABLE 1). Residue 501 is one of the key sites within the RBD involved in ACE2 binding $2,178,179$, and recent preliminary reports demonstrate that N501Y mutation strengthens RBD interaction with hACE2 $\left(\mathrm{REFS}^{180,181}\right)$ and increases the infectivity and virulence of variants containing the mutation ${ }^{182-184}$. Interestingly, data indicate that the N501Y substitution also lends the $S$ protein the ability to utilize mouse and rat ACE2 orthologues ${ }^{182,185,186}$, raising concern for the potential of new rodent reservoirs. A recent variant, Delta (B.1.617.2), first identified in India, does not have this N501Y mutation, but it nonetheless exhibits significantly increased transmissibility by an unknown mechanism ${ }^{187}$. Increased cleavage at the S1-S2 boundary due to the P681R mutation in the furin-cleavage site might further contribute to increased transmissibility ${ }^{128,188}$.

Immune escape. Viruses escape immunity by mutating the residues recognized by neutralizing antibodies. As immune escape is necessary only in the presence of immune pressure, no escape variants appeared in the early days of the pandemic, with lower dissemination and in the absence of vaccination. In recent months, however, several neutralization-resistant variants have emerged. The aforementioned Alpha, Beta, Gamma and Delta variants and the Epsilon variant (B.1.429, California lineage) exhibit decreased sensitivity to neutralization by immune plasma derived from convalescent patients with COVID-19 or vaccinated individuals ${ }^{40-45,185,189-194}$. The possibility that these recent variants emerged from the infection of previously infected or vaccinated individuals is supported by studies in which escape variants were experimentally generated in the presence of neutralizing antibodies, convalescent sera or sera derived from vaccinated individuals ${ }^{195-198}$. In these studies, the same mutations, including K417N, E484K and N501Y in the RBD, which are the hallmarks of the Alpha, Beta, and Gamma variants, were identified. These variants are also fully or partially resistant to the therapeutic monoclonal antibodies bamlanivimab (Eli Lilly) and/or casirivimab (Regeneron) $)^{178,189,197}$. It is likely that other variants, Eta (B.1.525, United Kingdom), Iota (B.1.526, New York) and Kappa (B.1.617.1, India), are also less sensitive to neutralization, owing to the Glu484 mutation to either Lys or Gln (E484K/Q). The rapidly spreading Delta variant does not carry E484K/Q, but it does have the additional mutation L452R, which confers neutralization resistance ${ }^{199}$ and is also present in the Eta and Iota variants.

\section{Conclusions and perspective}

Three zoonotic coronaviruses have emerged to cause severe disease in humans in the last two decades: SARS-CoV, MERS-CoV and SARS-CoV-2. The frequency of these zoonoses, accelerated by increased human intrusion into previously undisturbed habitats, suggests that new coronaviruses and other emerging pathogens will continue to threaten human health. Despite the warning signs from SARS-CoV and MERS-CoV outbreaks, few anticipated a pandemic of the scale that was caused by SARS-CoV-2. Fortunately, and owing in part to lessons learned from the previous outbreaks, our understanding of SARS-CoV-2 biology and the development of vaccines (BOX 2; TABLE 2) and therapeutics have proceeded at an unprecedented pace. In spite of these achievements, there remain many outstanding questions whose answers may assist in the development of new tools to control these viruses.

One question is when in its natural history did the virus acquire a furin site? As already mentioned, cleavage of the SARS-CoV-2 S protein into the $S 1$ and $S 2$ subunits results in an unstable $S$ protein. Nonetheless, the virus has judiciously held onto the furin site throughout the pandemic without deleting or mutating it. In addition, this furin site was shown to be important for transmission in ferrets ${ }^{128}$. One logical explanation is that the cleavage of the ancestral sequence at the S1-S2 junction (the sequence before the virus acquired the furin site) by target-cell proteases such as TMPRSS2 might have been inefficient, and thus the virus opted to precleave the site at the cost of resulting $S$ protein instability. On the other hand, studies show that SARS-CoV-2 is nonetheless more dependent on TMPRSS2 than on cathepsins ${ }^{138}$. Recent studies on IFITM proteins, type I interferon-induced endosomal virus restriction factors, provide one clue 
Box 2 | Vaccines against SARS-CoV-2

Vaccine products against SARS-CoV-2 based on several technology platforms (see the figure) have been advanced to clinical trials and emergency use worldwide (TABLE 2). In a conventional approach, inactivated whole-virus vaccine (far left in the figure) is generated by inactivating purified SARS-CoV-2 with formaldehyde and mixing it with an adjuvant such as alum (CoronaVac) or Algel-IMDG (BBV152) ${ }^{217,222}$. Whereas the spike (S) protein is the sole source of T cell epitopes in most other popular SARS-CoV-2 vaccines, whole-virus vaccine provides a wide repertoire of T cell epitopes in other viral proteins. The disadvantages, however, include lower immunogenicity owing to the perturbation of the antigen structure by the fixation process, production difficulties and residual risks, albeit minor, associated with incomplete inactivation of the virus.

The protein subunit-nanoparticle vaccine (middle left in the figure) is produced by incorporation of purified recombinant S protein into polysorbate 80 micelles with the addition of the saponin-based adjuvant Matrix-M (NVX-CoV2373) ${ }^{224}$. Preliminary trial results for NVX-CoV2373 demonstrate robust humoral and cell-mediated immunity, although the induction of the latter by Matrix-M is not yet completely understood ${ }^{260}$.

Two vaccine platforms using gene therapy technologies to elicit production of the $S$ protein antigen in host cells have also proven effective and have exceptional intrinsic immunogenicity. These are non-replicating recombinant adenoviral vector systems (middle right in the figure), which carry the gene encoding the S protein (Gam-COVID-Vac, AZD1222 and Ad26.COV2.S) ${ }^{219,221,223}$ and mRNA-LNP (lipid nanoparticle) systems (far right in the figure), wherein chemically modified mRNA encoding the $S$ protein bound to ionizable lipids is encapsulated inside a layer of mixed lipids (BNT162b2 and mRNA-1273) $)^{218,220}$. The response to the COVID-19 pandemic has highlighted distinct advantages of the mRNA-LNP technology in rapid prototyping and manufacturing on a large scale ${ }^{261}$.

All current vaccines are based on the S protein sequence of the Wuhan-Hu-1 strain predating the D614G mutation, which stabilizes the $S$ protein ${ }^{27,127,171,174}$ and is present in all currently circulating variants (TABLE 1). To stabilize the $S$ protein, some vaccine designs incorporated furin-site knockout and/or diproline mutations in the S protein. 'Diproline mutation' refers to the mutation of two consecutive residues in the S2 subunit (Lys986 and Val987) to proline and used to stabilize prefusion conformation of the S protein ${ }^{21}$. Other vaccines, such as AZD1222 and the inactivated whole-virus vaccines, utilize the unmodified S protein ${ }^{217,221,222}$ (TABLE 2). Therefore, differences in vaccine efficacy may be due to not only their differing vectors and formulations but also the particular $\mathrm{S}$ protein construct used.

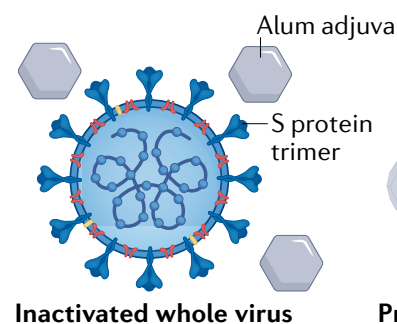

Inactivated whole virus

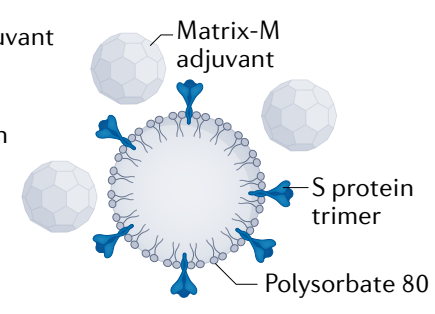

Protein subunit-nanoparticle

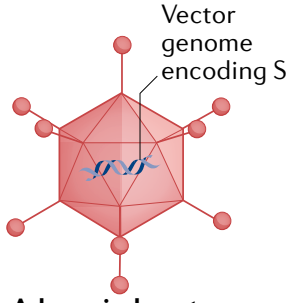

Adenoviral vector

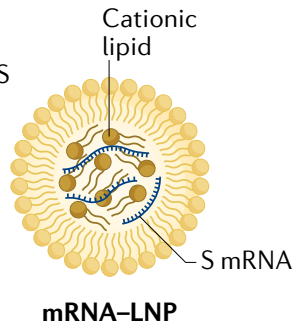

mRNA-LNP to this puzzle. SARS-CoV-2 lacking the polybasic site is more sensitive to IFITM-mediated restriction than the wild-type virus ${ }^{128,149}$, suggesting the virus chose to use TMPRSS2 to enter cells to avoid IFITM-mediated restriction in the endosome. Alternatively, this preference of SARS-CoV-2 for TMPRSS2 can also be explained if tight folding of the SARS-CoV S protein allows the precise exposure of the $\mathrm{S} 2$ ' site to cathepsin L, a protease with low substrate specificity, while the difference in SARS-CoV-2 S protein folding may not confine cathepsin digestion to only the S2' site, leading to overdigestion of the neighbouring sequences, which renders the fusion peptide non-functional. These situations would force SARS-CoV-2 to prefer TMPRSS2 to cathepsins. If so, the furin dependency and TMPRSS2 dependency of SARS-CoV-2 appear to reflect a choice of less bad options. Of these, S protein instability was compensated by acquisition of the D614G mutation ${ }^{27,127}$. If TMPRSS2 inhibitors become widely used, it is possible the virus will acquire mutations that facilitate escape from inhibitory action of IFITM proteins and enable more precise use of cathepsins, suggesting the benefit of use of combinatorial therapies targeting both pathways (camostat mesylate plus hydroxychloroquine).

Some critical questions are associated with the link between transmissibility and disease severity. As a virus better adapts in a species, the severity of the disease can diminish, but recent changes in SARS-CoV-2 have increased both transmissibility and hospitalizations. A positive relationship between transmissibility and hospitalizations may reflect a common underlying mechanism: higher affinity for ACE2 can increase both. More efficient binding to ACE2 can promote replication in the upper respiratory tract, promoting more efficient transmission, and it can also increase replication in the lower respiratory tract and systemically, causing severer disease. One outstanding question is whether the $S$ protein has reached the maximum affinity for hACE2 through RBD mutations such as N501Y or whether it will further mutate and continue to enhance both transmissibility and pathogenicity. Or might countervailing selection pressures result in transmission gains but with milder disease? If the differences between the upper and lower respiratory tracts allow the virus to adapt specifically to, say, nasal epithelial cells, the link between transmission and disease severity may be broken.

At the time of writing, the pandemic appears to be shifting gears, moving from an early period of adaptation of the virus to its new human host to a longer period where immune escape will shape $S$ protein evolution. A key question here is what future vaccine antigens should look like. The $S$ protein is rapidly diversifying, and current vaccination strategies (BOX 2; TABLE 2) may soon become impractical, necessitating vaccine deployment against 
Table 2 | Selected products used in global vaccination campaigns

\begin{tabular}{|c|c|c|c|c|c|}
\hline Product & Developers & Platform & Antigen & First deployment & Ref. \\
\hline CoronaVac & Sinovac & $\begin{array}{l}\text { Inactivated virus (alum } \\
\text { adjuvant) }\end{array}$ & $\begin{array}{l}\text { Whole virion, } \\
\text { including WT }^{\text {Sprotein }}\end{array}$ & $\begin{array}{l}29 \text { Aug } 2020 \\
\text { (China) }\end{array}$ & 217 \\
\hline $\begin{array}{l}\text { BNT162b2 } \\
\text { (Comirnaty) }\end{array}$ & Pfizer, BioNTech & mRNA-lipid nanoparticle & S protein $(2 \mathrm{P})^{\mathrm{b}}$ & $\begin{array}{l}2 \text { Dec } 2020 \text { (United } \\
\text { Kingdom) }\end{array}$ & 218 \\
\hline $\begin{array}{l}\text { Gam-COVID-Vac } \\
\text { (Sputnik V) }\end{array}$ & $\begin{array}{l}\text { Gamaleya Research } \\
\text { Institute, Health } \\
\text { Ministry of the } \\
\text { Russian Federation }\end{array}$ & $\begin{array}{l}\text { Non-replicating } \\
\text { adenoviral vectors rAd26 } \\
\text { and rAd5 }\end{array}$ & WT S protein ${ }^{a}$ & 5 Dec 2020 (Russia) & 219 \\
\hline mRNA-1273 & $\begin{array}{l}\text { Moderna, US National } \\
\text { Institute of Allergy and } \\
\text { Infectious Diseases }\end{array}$ & mRNA-lipid nanoparticle & S protein $(2 \mathrm{P})^{\mathrm{b}}$ & $\begin{array}{l}18 \text { Dec } 2020 \\
\text { (United States) }\end{array}$ & 220 \\
\hline $\begin{array}{l}\text { AZD1222 } \\
\text { (Covishield) }\end{array}$ & $\begin{array}{l}\text { AstraZeneca, University } \\
\text { of Oxford }\end{array}$ & $\begin{array}{l}\text { Non-replicating } \\
\text { adenoviral vector } \\
\text { ChAdOx1 }\end{array}$ & WT S protein & $\begin{array}{l}30 \text { Dec } 2020 \\
\text { (United Kingdom) }\end{array}$ & 221 \\
\hline $\begin{array}{l}\text { BBV152 } \\
\text { (Covaxin) }\end{array}$ & $\begin{array}{l}\text { Bharat Biotech } \\
\text { International }\end{array}$ & $\begin{array}{l}\text { Inactivated virus } \\
\text { (Algel-IMDG adjuvant) }\end{array}$ & $\begin{array}{l}\text { Whole virion, } \\
\text { including WT } \\
\text { S protein }\end{array}$ & 2 Jan 2021 (India) & 222 \\
\hline Ad26.COV2.S & $\begin{array}{l}\text { Janssen Pharmaceutical } \\
\text { (Johnson \& Johnson) }\end{array}$ & $\begin{array}{l}\text { Non-replicating } \\
\text { adenoviral vector rAd26 }\end{array}$ & $\begin{array}{l}\text { Sprotein } \\
(\mathrm{FKO}+2 \mathrm{P})^{\mathrm{c}}\end{array}$ & $\begin{array}{l}17 \text { Feb } 2021 \text { (South } \\
\text { Africa) }\end{array}$ & 223 \\
\hline NVX-CoV2373 & Novavax & $\begin{array}{l}\text { Protein subunit- } \\
\text { nanoparticle (Matrix M } \\
\text { adjuvant) }\end{array}$ & $\begin{array}{l}\text { S protein } \\
(\mathrm{FKO}+2 \mathrm{P})^{\mathrm{c}}\end{array}$ & Pending $^{d}$ & 224 \\
\hline
\end{tabular}

The vaccines that had received or are close to receiving emergency use authorization at the time of writing are included. S protein, spike protein; 2P, diproline mutation (K986P and V987P); FKO, furin-cleavage site knocked out; WT, wild type. aFull-length WT S protein. ${ }^{\circ}$ Full-length S protein with $2 \mathrm{P}$ mutation in the S2 subunit to stabilize prefusion conformation. ${ }^{\mathrm{C}}$ Full-length $\mathrm{S}$ protein with FKO and $2 \mathrm{P}$ mutation added to stabilize prefusion conformation. ${ }^{\mathrm{d} A p p l i c a t i o n}$ for emergency use authorization had been submitted in several countries as of August 2021 but had not been received at the time of writing.

every major circulating variant. The major antibodyneutralizing epitope of the S protein is the RBD, accounting for more than $90 \%$ of all neutralizing activity, although the NTD also has some neutralizing epitopes. Importantly, the NTD is changing faster, acquiring more mutations and deletions than the RBD; understandably because the NTD plays at most a secondary role in viral entry and thus is subject to fewer constraints on its evolution. The remainder of the S protein, including the S2 subunit, does not appear to elicit significant neutralizing responses. Therefore, RBD vaccines presenting multiple RBDs derived from various escape variants may make superior antigens for future vaccines.

A related question is whether the accessible pathways of viral escape are essentially unlimited, or whether they are constrained, especially in the RBD, so that only a tractable number of mutations are likely to emerge. If the latter is true, they might be anticipated and blocked pre-emptively. So far, four major regions or residues in the $S 1$ subunit have been identified that render the virus less sensitive to immune sera: a supersite in the NTD (residues 14-26, 141-156 and 246-260) 200-203 $^{20}$ and residues 417,484 and 501 in the $\mathrm{RBD}^{40,200,204}$. Interestingly, the immune-escape mutations selected in several in vitro studies overlap with those that naturally emerged from human infection ${ }^{40,191,202,205}$ and those selected in the presence of monoclonal antibodies or sera derived from infected or vaccinated individuals ${ }^{195-197}$. These observations imply that there might indeed be only a limited number of escape pathways the virus can take and that those pathways could be blocked. Alternatively, the mutations the virus has been accumulating so far could represent the first 'low-hanging fruit' available to the virus. Indeed, all major mutations in the RBD to date have been accessed through a single nucleotide change, and so, in the pessimistic scenario, the virus could further evolve through more complex mutations such as several synergistic or compensatory mutations in parallel. Moreover, several recent mutations have increased RBD affinity for ACE2 (TABLE 1). Unfortunately, this adaptation provides the virus with some breathing room to accommodate immune-escape mutations that will likely decrease the affinity for ACE2.

Thus, unfortunately, SARS-CoV-2 variants may continue to adapt in the human population for years or decades. This raises additional questions. As coronaviruses are especially good at recombining with other coronaviruse $^{206}$, will a recombination event with one of the milder coronaviruses that regularly circulate among humans generate an entirely new virus? Will SARS-CoV-2 diversify into even more distinct lineages more akin to the many forms of influenza A viruses with which we regularly contend? Will SARS-CoV-2 stably remain among our livestock or pests, perhaps creating reservoirs for new zoonoses? Perhaps the most critical question is whether we can finally learn the clearest lesson of the COVID-19 pandemic; namely, can we fully appreciate that viral infections are a major threat to all of us, but at the same time are fully addressable with our current technologies and effective implementation of well-established public health principles? If we learn this lesson well, hopefully this will be our last pandemic.

Published online 5 October 2021 
1. Lu, R. et al. Genomic characterisation and epidemiology of 2019 novel coronavirus: implications for virus origins and receptor binding. Lancet 395, 565-574 (2020).

2. Shang, J. et al. Structural basis of receptor recognition by SARS-CoV-2. Nature 581, 221-224 (2020).

Together with Lan, J. et al., this study provides one of two early crystal structures of the SARS-CoV-2 RBD-ACE2 complex, showing how the $S$ protein recognizes its receptor

3. Zhou, P. et al. A pneumonia outbreak associated with a new coronavirus of probable bat origin. Nature $\mathbf{5 7 9}$ 270-273 (2020).

4. Walls, A. C. et al. Structure, function, and antigenicity of the SARS-CoV-2 spike glycoprotein. Cell https:// doi.org/10.1016/j.cell.2020.02.058 (2020).

5. Lan, J. et al. Structure of the SARS-CoV-2 spike receptor-binding domain bound to the ACE2 receptor. Nature 581, 215-220 (2020).

Together with Shang, J. et al., this study provides one of two early crystal structures of the SARS-CoV-2 RBD-ACE2 complex, revealing how the $S$ protein recognizes its receptor.

6. Wang, Q. et al. Structural and functional basis of SARS-CoV-2 entry by using human ACE2. Cell 181, 894-904 (2020)

7. Li, W. et al. Angiotensin-converting enzyme 2 is a functional receptor for the SARS coronavirus. Nature 426, 450-454 (2003).

This study identifies ACE2 as the receptor for SARS-CoV.

8. Hofmann, H. et al. Human coronavirus NL63 employs the severe acute respiratory syndrome coronavirus receptor for cellular entry. Proc. Natl Acad. Sci. USA 102, 7988-7993 (2005).

9. Hoffmann, M., Kleine-Weber, H. \& Pöhlmann, S. A multibasic cleavage site in the spike protein of SARSCoV-2 is essential for infection of human lung cells. Mol. Cell 78, 779-784 (2020).

This article provides insight into the function and necessity of the S1-S2 furin-cleavage site for SARS-CoV-2 infection of human lung cells in vitro.

10. Shang J. et al. Cell entry mechanisms of SARS-CoV-2. Proc. Natl Acad. Sci. USA 117, 11727-11734 (2020).

11. Fehr, A. R. \& Perlman, S. Coronaviruses: an overview of their replication and pathogenesis. Methods Mol. Biol. 1282, 1-23 (2015).

12. Glowacka, I. et al. Evidence that TMPRSS2 activates the severe acute respiratory syndrome coronavirus spike protein for membrane fusion and reduces viral control by the humoral immune response. J. Virol. 85 4122-4134 (2011)

13. Matsuyama, S. et al. Efficient activation of the severe acute respiratory syndrome coronavirus spike protein by the transmembrane protease TMPRSS2. J. Virol. 84, 12658-12664 (2010).

This study and those by Glowacka et al. (2011) and Shulla et al. (2011) are the first to demonstrate the importance of TMPRSS2 in SARS-CoV infection.

14. Shulla, A. et al. A transmembrane serine protease is linked to the severe acute respiratory syndrome coronavirus receptor and activates virus entry. J. Virol. 85, 873-882 (2011).

15. Huang, I. C. et al. SARS coronavirus, but not human coronavirus NL63, utilizes cathepsin L to infect ACE2expressing cells. J. Biol. Chem. 281, 3198-3203 (2006).

16. Simmons, G. et al. Inhibitors of cathepsin L prevent severe acute respiratory syndrome coronavirus entry. Proc. Natl Acad. Sci. USA 102, 11876-11881 (2005). This study and that by Huang et al. (2006) are the first to identify the role of cathepsin $L$ in processing of the SARS-CoV S protein.

17. Bayati, A., Kumar, R., Francis, V. \& McPherson, P. S SARS-CoV-2 infects cells following viral entry via clathrin-mediated endocytosis. J. Biol. Chem. https:// doi.org/10.1016/j.jbc.2021.100306 (2021).

18. Inoue, Y. et al. Clathrin-dependent entry of severe acute respiratory syndrome coronavirus into target cells expressing ACE2 with the cytoplasmic tail deleted. J. Virol. 81, 8722-8729 (2007).

19. Watanabe, Y., Allen, J. D., Wrapp, D., McLellan, J. S $\&$ Crispin, M. Site-specific glycan analysis of the SARS-CoV-2 spike. Science 369, 330-333 (2020).

20. Harrison, S. C. Viral membrane fusion. Virology 479-480, 498-507 (2015)

21. Wrapp, D. et al. Cryo-EM structure of the 2019-nCoV spike in the prefusion conformation. Science 367 1260-1263 (2020).

Together with Walls et al. (2020), this study shows cryo-EM structures of the stabilized ectodomain $\mathrm{S}$ trimer of SARS-CoV-2, providing molecular insights into its membrane fusion machinery.
22. Gobeil, S. M. et al. D614G mutation alters SARS-CoV-2 spike conformation and enhances protease cleavage at the S1/S2 junction. Cell Rep. 34, 108630 (2021).

23. Yan, R. et al. Structural basis for the recognition of SARS-CoV-2 by full-length human ACE2. Science 367. 1444-1448 (2020).

24. Xia, S. et al. Inhibition of SARS-CoV-2 (previously 2019-nCoV) infection by a highly potent pancoronavirus fusion inhibitor targeting its spike protein that harbors a high capacity to mediate membrane fusion. Cell Res. 30, 343-355 (2020).

25. Cai, Y. et al. Distinct conformational states of SARSCoV-2 spike protein. Science $369,1586-1592$ (2020).

This study provides the first cryo-EM structure of the unmodified full-length S protein of SARS-CoV-2 in both the prefusion conformation and the postfusion conformation.

26. Bangaru, S. et al. Structural analysis of full-length SARS-CoV-2 spike protein from an advanced vaccine candidate. Science 370, 1089-1094 (2020).

27. Zhang, J. et al. Structural impact on SARS-CoV-2 spike protein by D614G substitution. Science 372 , 525-530 (2021)

This study provides the cryo-EM structure of the unmodified full-length SARS-CoV-2 S protein with the D614G mutation, supporting its role in stabilizing $\mathbf{S} 1-\mathrm{S} 2$ association.

28. Turonova, B. et al. In situ structural analysis of SARS CoV-2 spike reveals flexibility mediated by three hinges. Science 370, 203-208 (2020).

29. Ke, Z. et al. Structures and distributions of SARS CoV-2 spike proteins on intact virions. Nature $\mathbf{5 8 8}$, 498-502 (2020).

This is one of the first reports on SARS-CoV-2 S trimers in situ on the virion surface imaged by cryo$\mathrm{EM}$ and cryo-electron tomography, revealing their high-resolution structure, conformational flexibility and distribution

30. Yao, H. et al. Molecular architecture of the SARS-CoV-2 virus. Cell 183, 730-738 (2020).

31. Liu, C et al. The architecture of inactivated SARS-CoV-2 with postfusion spikes revealed by cryo-EM and cryo-ET. Structure 28, 1218-1224 (2020)

32. Kunkel, F. \& Herrler, G. Structural and functional analysis of the surface protein of human coronavirus OC43. Virology 195, 195-202 (1993).

33. Schultze, B., Gross, H. J., Brossmer, R. \& Herrler, G The $\mathrm{S}$ protein of bovine coronavirus is a hemagglutinin recognizing 9-O-acetylated sialic acid as a receptor determinant. J. Virol. 65, 6232-6237 (1991).

34. Krempl, C., Schultze, B., Laude, H. \& Herrler, G Point mutations in the $\mathrm{S}$ protein connect the sialic acid binding activity with the enteropathogenicity of transmissible gastroenteritis coronavirus. J. Virol. 71 , 3285-3287 (1997).

35. Zhou, $\mathrm{H}$. et al Structural definition of a neutralization epitope on the $\mathrm{N}$-terminal domain of MERS-CoV spike glycoprotein. Nat. Commun. 10, 3068 (2019).

36. Chi, X. et al. A neutralizing human antibody binds to the $\mathrm{N}$-terminal domain of the Spike protein of SARS-CoV-2. Science 369, 650-655 (2020). This is one of the first reports showing that the RBD and the NTD are the two major neutralizing targets on the SARS-CoV-2 S trimer.

37. Liu, L. et al. Potent neutralizing antibodies against multiple epitopes on SARS-CoV-2 spike. Nature $\mathbf{5 8 4}$ 450-456 (2020)

38. Wibmer, C. K. et al. SARS-CoV-2 501Y.V2 escapes neutralization by South African COVID-19 donor plasma. Nat. Med. 27, 622-625 (2021)

39. Wu, K. et al. Serum neutralizing activity elicited by mRNA-1273 vaccine. N. Engl. J. Med. 384 1468-1470 (2021).

40. Wang, Z. et al. mRNA vaccine-elicited antibodies to SARS-CoV-2 and circulating variants. Nature https:/ doi.org/10.1038/s41586-021-03324-6 (2021).

41. Wang, P. et al. Antibody resistance of SARS-CoV-2 variants B.1.351 and B.1.1.7. Nature https://doi.org/ 10.1038/s41586-021-03398-2 (2021)

42. Edara, V. V. et al. Infection and vaccine-induced neutralizing-antibody responses to the SARS-CoV-2 B. 1.617 variants. N. Engl. J. Med. https://doi.org/ 10.1056/NEJMc2107799 (2021)

43. Wall, E. C. et al. Neutralising antibody activity against SARS-CoV-2 VOCs B.1.617.2 and B.1.351 by BNT162b2 vaccination. Lancet 397, 2331-2333 (2021).

44. Planas, D. et al. Reduced sensitivity of SARS-CoV-2 variant Delta to antibody neutralization. Nature https://doi.org/10.1038/s41586-021-03777-9 (2021).
45. Sheikh, A. et al. SARS-CoV-2 Delta VOC in Scotland: demographics, risk of hospital admission, and vaccine effectiveness. Lancet 397, 2461-2462 (2021).

46. Grabowski, F., Preibisch, G., Gizinski, S. Kochanczyk, M. \& Lipniacki, T. SARS-CoV-2 variant of concern 202012/01 has about twofold replicative advantage and acquires concerning mutations. Viruses https://doi.org/10.3390/v13030392 (2021).

47. Tegally, $\mathrm{H}$. et al. Detection of a SARS-CoV- 2 variant of concern in South Africa. Nature 592, 438-443 (2021).

48. Voloch, C. M. et al. Genomic characterization of a novel SARS-CoV-2 lineage from Rio de Janeiro, Brazil. J. Virol. https://doi.org/10.1128/JVI.00119-21 (2021).

49. Tada, T. et al. SARS-CoV-2 lambda variant remains susceptible to neutralization by mRNA vaccine-elicited antibodies and convalescent serum. Preprint at bioRxiv https://doi.org/10.1101/2021.07.02.450959 (2021).

50. Li, F., Li, W., Farzan, M. \& Harrison, S. C. Structure of SARS coronavirus spike receptor-binding domain complexed with receptor. Science 309, 1864-1868 (2005).

This study provides the crystal structure of the SARS-CoV RBD bound to ACE2, illuminating an interface shared by SARS-CoV-2.

51. Wells, H. L. et al. The evolutionary history of ACE2 usage within the coronavirus subgenus Sarbecovirus. Virus Evol. 7, veab007 (2021).

52. Tong, P. et al. Memory B cell repertoire for recognition of evolving SARS-CoV-2 spike. Cell https://doi.org/ 10.1016/j.cell.2021.07.025 (2021).

53. Hansen, J et al. Studies in humanized mice and convalescent humans yield a SARS-CoV-2 antibody cocktail. Science 369, 1010-1014 (2020).

54. Robbiani, D. F. et al. Convergent antibody responses to SARS-CoV-2 in convalescent individuals. Nature 584, 437-442 (2020)

55. Pinto, D. et al. Cross-neutralization of SARS-CoV-2 by a human monoclonal SARS-CoV antibody. Nature 583, 290-295 (2020).

56. Yuan, M. et al. A highly conserved cryptic epitope in the receptor binding domains of SARS-CoV-2 and SARS-CoV. Science 368, 630-633 (2020)

57. Walls, A. C. et al. Tectonic conformational changes of a coronavirus spike glycoprotein promote membrane fusion. Proc. Natl Acad. Sci. USA 114, 11157-11162 (2017).

58. Fan, X., Cao, D., Kong, L. \& Zhang, X. Cryo-EM analysis of the post-fusion structure of the SARS-CoV spike glycoprotein. Nat. Commun. 11, 3618 (2020).

59. Turner, A. J. \& Hooper, N. M. The angiotensinconverting enzyme gene family: genomics and pharmacology. Trends Pharmacol. Sci. 23, 177-183 (2002).

60. Donoghue, $\mathrm{M}$. et al. A novel angiotensin-converting enzyme-related carboxypeptidase (ACE2) converts angiotensin I to angiotensin 1-9. Circ. Res. 87, E1-E9 (2000).

61. Crackower, M. A. et al. Angiotensin-converting enzyme 2 is an essential regulator of heart function. Nature 417, 822-828 (2002)

62. Tipnis, S. R. et al. A human homolog of angiotensinconverting enzyme. Cloning and functional expression as a captopril-insensitive carboxypeptidase. J. Biol. Chem. 275, 33238-33243 (2000).

63. Lu, G. et al. Molecular basis of binding between novel human coronavirus MERS-CoV and its receptor CD26. Nature 500, 227-231 (2013).

64. Yeager, C. L. et al. Human aminopeptidase $\mathrm{N}$ is a receptor for human coronavirus 229E. Nature 357 420-422 (1992).

65. Raj, V. S. et al. Dipeptidyl peptidase 4 is a functional receptor for the emerging human coronavirus-EMC. Nature 495, 251-254 (2013).

66. Li, W. et al. Receptor and viral determinants of SARScoronavirus adaptation to human ACE2. EMBO J. 24 , 1634-1643 (2005).

67. Kuba, K. et al. A crucial role of angiotensin converting enzyme 2 (ACE2) in SARS coronavirus-induced lung injury. Nat. Med. 11, 875-879 (2005).

68. Zhou, T. et al. Cryo-EM structures of SARS-CoV-2 spike without and with ACE2 reveal a pH-dependent switch to mediate endosomal positioning of receptor-binding domains. Cell Host Microbe 28, 867-879 e865 (2020).

69. Xiao, T. et al. A trimeric human angiotensin-converting enzyme 2 as an anti-SARS-CoV-2 agent. Nat. Struct. Mol. Biol. 28, 202-209 (2021).

70. Song, W., Gui, M., Wang, X. \& Xiang, Y. Cryo-EM structure of the SARS coronavirus spike glycoprotein in complex with its host cell receptor ACE2. PLOS Pathog. 14, e1007236 (2018). 
71. Hou, Y. J. et al. SARS-CoV-2 reverse genetics reveals a variable infection gradient in the respiratory tract. Cell 182, 429-446 (2020).

This study shows that SARS-CoV-2 infection levels in COVID-19 autopsied lungs corresponds to a gradient of ACE2 expression in the upper and lower airways.

72. Sungnak, W. et al. SARS-CoV-2 entry factors are highly expressed in nasal epithelial cells together with innate immune genes. Nat. Med. 26, 681-687 (2020).

73. Wang, Y. et al. A comprehensive investigation of the mRNA and protein level of ACE2, the putative receptor of SARS-CoV-2, in human tissues and blood cells. Int. J. Med. Sci. 17, 1522-1531 (2020).

74. Zou, X. et al. Single-cell RNA-seq data analysis on the receptor ACE2 expression reveals the potential risk of different human organs vulnerable to 2019-nCoV infection. Front. Med. 14, 185-192 (2020).

75. Ahn, J. H. et al. Nasal ciliated cells are primary targets for SARS-CoV-2 replication in early stage of COVID-19. J. Clin. Invest. https://doi.org/10.1172/JCI 148517 (2021).

76. Lee, I. T. et al. ACE2 localizes to the respiratory cilia and is not increased by ACE inhibitors or ARBs. Nat. Commun. https://doi.org/10.1038/s41467-02019145-6 (2020).

\section{This histological study of human donor tissues} clarifies ACE2 tissue distribution and establishes that antihypertensive drugs do not potentiate SARS-CoV-2 infection

77. Ye, M. H. et al. Increased ACE 2 and decreased ACE protein in renal tubules from diabetic mice - a renoprotective combination? Hypertension $\mathbf{4 3}$ 1120-1125 (2004)

78. Lindner, D. et al. Association of cardiac infection with SARS-CoV-2 in confirmed COVID-19 autopsy cases. JAMA Cardiol. 5, 1281-1285 (2020).

79. Zhuang, M.-W. et al. Increasing host cellular receptorangiotensin-converting enzyme 2 expression by coronavirus may facilitate 2019-nCoV (or SARS-CoV-2) infection. J. Med. Virol. 92, 2693-2701 (2020).

80. Smith, J. C et al. Cigarette smoke exposure and inflammatory signaling increase the expression of the SARS-CoV-2 receptor ACE2 in the respiratory tract. Dev. Cell 53, 514-529 (2020)

81. Ziegler, C. G. K. et al. SARS-CoV-2 receptor ACE2 Is an interferon-stimulated gene in human airway epithelial cells and is detected in specific cell subsets across tissues. Cell 181, 1016-1035 (2020).

82. Onabajo, O. O. et al. Interferons and viruses induce a novel truncated ACE2 isoform and not the full-length SARS-CoV-2 receptor. Nat. Genet. 52, 1283-1293 (2020).

83. Baker, S. A., Kwok, S., Berry, G. J. \& Montine, T. J. Angiotensin-converting enzyme 2 (ACE2) expression increases with age in patients requiring mechanical ventilation. PLOS ONE 16, e0247060 (2021).

84. Muus, C. et al. Single-cell meta-analysis of SARS-CoV-2 entry genes across tissues and demographics. Nat. Med. 27, 546-559 (2021).

85. Chen, J. et al. Individual variation of the SARS-CoV-2 receptor ACE2 gene expression and regulation. Aging Cell https://doi.org/10.1111/acel.13168 (2020).

86. Patanavanich, R. \& Glantz, S. A. Smoking is associated with COVID-19 progression: a meta-analysis. Nicotine Tob. Res. 22, 1653-1656 (2020)

87. Zhao, Q. et al. The impact of COPD and smoking history on the severity of COVID-19: a systemic review and meta-analysis. J. Med. Virol. 92, 1915-1921 (2020).

88. Jacobs, M. et al. Increased expression of ACE2 the SARS-CoV-2 entry receptor, in alveolar and bronchial epithelium of smokers and COPD subjects. Eur. Respir. J. https://doi.org/10.1183 13993003.02378-2020 (2020)

89. Leung, J. M. et al. ACE-2 expression in the small airway epithelia of smokers and COPD patients: implications for COVID-19. Eur. Respir. J. https:// doi.org/10.1183/13993003.00688-2020 (2020).

90. Rossato, M. et al. Current smoking is not associated with COVID-19. Eur. Respir. J. https://doi.org/10.1183/ 13993003.01290-2020 (2020)

91. Williamson, E. J. et al. Factors associated with COVID-19-related death using OpenSAFELY. Nature 584, 430-436 (2020).

92. Peters, M. C. et al. COVID-19-related genes in sputum cells in asthma. Relationship to demographic features and corticosteroids. Am. J. Respir. Crit. Care Med. 202, 83-90 (2020)

93. Furuhashi, M. et al. Urinary angiotensin-converting enzyme 2 in hypertensive patients may be increased by olmesartan, an angiotensin II receptor blocker Am. J. Hypertens. 28, 15-21 (2015).
94. Fang, L., Karakiulakis, G. \& Roth, M. Are patients with hypertension and diabetes mellitus at increased risk for COVID-19 infection? Lancet Respir. Med. 8, e21 (2020).

95. Patel, A. B. \& Verma, A. COVID-19 and angiotensinconverting enzyme inhibitors and angiotensin receptor blockers: what is the evidence? JAMA 323 1769-1770 (2020)

96. Mancia, G., Rea, F., Ludergnani, M., Apolone, C. \& Corrao, G. Renin-angiotensin-aldosterone system blockers and the risk of Covid-19. N. Engl. J. Med. 382, 2431-2440 (2020).

97. Reynolds, H. R. et al. Renin-angiotensin-aldosterone system inhibitors and risk of Covid-19. N. Engl. J. Med. 382, 2441-2448 (2020)

98. Mou, H. et al. Mutations derived from horseshoe bat ACE2 orthologs enhance ACE2-Fc neutralization of SARS-CoV-2. PLoS Pathog. 17, e1009501 (2021).

99. Guan, Y. et al. Isolation and characterization of viruses related to the SARS coronavirus from animals in southern China. Science 302, 276-278 (2003).

100. Lam, T. T. et al. Identifying SARS-CoV-2-related coronaviruses in Malayan pangolins. Nature 583 282-285 (2020)

101. Liu, P. et al. Are pangolins the intermediate host of the 2019 novel coronavirus (SARS-CoV-2)? PLoS Pathog. 16, e1008421 (2020).

102. Xiao, K. et al. Isolation of SARS-CoV-2-related coronavirus from Malayan pangolins. Nature $\mathbf{5 8 3}$ 286-289 (2020)

103. Peiris, J. S., Guan, Y. \& Yuen, K. Y. Severe acute respiratory syndrome. Nat. Med. 10, S88-S97 (2004).

104. Zhou, H. et al. A novel bat coronavirus closely related to SARS-CoV-2 contains natural insertions at the S1/S2 cleavage site of the spike protein. Curr. Biol. 30, 2196-2203 e2193 (2020).

105. Yan, $\mathrm{H}$. et al. ACE2 receptor usage reveals variation in susceptibility to SARS-CoV and SARS-CoV-2 infection among bat species. Nat. Ecol. Evol. https://doi.org/ 10.1038/s41559-021-01407-1 (2021)

106. Liu, Y. et al. Functional and genetic analysis of viral receptor ACE2 orthologs reveals a broad potential host range of SARS-CoV-2. Proc. Natl Acad. Sci. USA https://doi.org/10.1073/pnas.2025373118 (2021).

107. Jeffers, S. A. et al. CD209L (L-SIGN) is a receptor for severe acute respiratory syndrome coronavirus. Proc. Natl Acad. Sci. USA 101, 15748-15753 (2004).

108. Yang, Z. Y. et al. pH-dependent entry of severe acute respiratory syndrome coronavirus is mediated by the spike glycoprotein and enhanced by dendritic cell transfer through DC-SIGN. J. Virol. 78, 5642-5650 (2004).

109. Amraie, R. et al. CD209L/L-SIGN and CD209/DC-SIGN act as receptors for SARS-CoV-2. ACS Cent. Sci. https://doi.org/10.1021/acscentsci.0c01537 (2021).

110. Khoo, U. S., Chan, K. Y., Chan, V. S. \& Lin, C. L. DC-SIGN and L-SIGN: the SIGNs for infection. J. Mol. Med. 86, 861-874 (2008).

111. Geijtenbeek, T. B. et al. Identification of DC-SIGN, a novel dendritic cell-specific ICAM-3 receptor that supports primary immune responses. Cell 100 575-585 (2000)

112. Ichimura, T. et al. KIM-1/TIM-1 is a receptor for SARS CoV-2 in lung and kidney. Preprint at medRxiv https:// doi.org/10.1101/2020.09.16.20190694 (2020).

113. Wang, S. et al. AXL is a candidate receptor for SARS CoV-2 that promotes infection of pulmonary and bronchial epithelial cells. Cell Res. 31, 126-140 (2021).

114. Amara, A. \& Mercer, J. Viral apoptotic mimicry. Nat. Rev. Microbiol. 13, 461-469 (2015).

115. Jemielity, S. et al. TIM-family proteins promote infection of multiple enveloped viruses through virionassociated phosphatidylserine. PLoS Pathog. 9 e1003232 (2013).

116. Richard, A. S. et al. AXL-dependent infection of human fetal endothelial cells distinguishes Zika virus from other pathogenic flaviviruses. Proc. Natl Acad. Sci. USA 114, 2024-2029 (2017)

117. Marzi, A. et al. DC-SIGN and DC-SIGNR interact with the glycoprotein of Marburg virus and the $S$ protein of severe acute respiratory syndrome coronavirus. J. Virol. 78, 12090-12095 (2004).

118. Chen, Z. et al. Function of HAb 18G/CD147 in invasion of host cells by severe acute respiratory syndrome coronavirus. J. Infect. Dis. 191, 755-760 (2005).

119. Wang, K. et al. CD147-spike protein is a novel route fo SARS-CoV-2 infection to host cells. Signal. Transduct. Target. Ther. 5, 283 (2020).

120. Costa, L. B. et al. Insights on SARS-CoV-2 molecular interactions with the renin-angiotensin system. Front. Cell Dev. Biol. 8, 559841 (2020).
121. Shilts, J., Crozier, T. W. M., Greenwood, E. J. D. Lehner, P. J. \& Wright, G. J. No evidence for basigin/ CD147 as a direct SARS-CoV-2 spike binding receptor. Sci. Rep. 11, 413 (2021).

122. Cantuti-Castelvetri, L. et al. Neuropilin- 1 facilitates SARS-CoV-2 cell entry and infectivity. Science $\mathbf{3 7 0}$, 856-860 (2020)

123. Daly, J. L. et al. Neuropilin- 1 is a host factor for SARSCoV-2 infection. Science 370, 861-865 (2020).

124. Zhen-Lu, L. \& Matthias, B. Neuropilin-1 assists SARS CoV- 2 infection by stimulating the separation of spike protein domains S1 and S2. Biophys. J. https://doi.org/ 10.1016/j.bpj.2021.05.026 (2021).

125. Camargo, S. M. et al. Tissue-specific amino acid transporter partners ACE2 and collectrin differentially interact with hartnup mutations. Gastroenterology 136, 872-882 (2009)

126. Belouzard, S., Chu, V. C. \& Whittaker, G. R. Activation of the SARS coronavirus spike protein via sequential proteolytic cleavage at two distinct sites. Proc. Natl Acad. Sci. USA 106, 5871-5876 (2009).

127. Zhang, L. et al. SARS-CoV-2 spike-protein D614C mutation increases virion spike density and infectivity. Nat. Commun. 11, 6013 (2020)

This article shows that enhanced infectivity of the SARS-CoV-2 variants harbouring the D614C $S$ protein mutation is due to a reduced level of premature $\mathrm{S} 1$ subunit shedding.

128. Peacock, T. P. et al. The furin cleavage site in the SARS-CoV-2 spike protein is required for transmission in ferrets. Nat. Microbiol. 6, 899-909 (2021). This study demonstrates in ferrets a critical in vivo role for the $\mathrm{S}$ protein furin-cleavage site in SARS-CoV-2 infection.

129. Limburg, H. et al. TMPRSS2 is the major activating protease of influenza a virus in primary human airway cells and influenza B virus in human type II pneumocytes. J. Virol. https://doi.org/10.1128/ JVI.00649-19 (2019)

130. Shen, L. W., Mao, H. J., Wu, Y. L., Tanaka, Y. \& Zhang, W. TMPRSS2: a potential target for treatment of influenza virus and coronavirus infections. Biochimie 142, 1-10 (2017).

131. Sakai, K. et al. TMPRSS2 independency for haemagglutinin cleavage in vivo differentiates influenza B virus from influenza A virus. Sci. Rep. 6 29430 (2016).

132. Szabo, R. \& Bugge, T. H. Type II transmembrane serine proteases in development and disease. Int. J. Biochem. Cell Biol. 40, 1297-1316 (2008).

133. Szabo, R. \& Bugge, T. H. Membrane-anchored serine proteases in vertebrate cell and developmental biology. Annu. Rev. Cell Dev. Biol. 27, 213-235 (2011).

134. Qi, F., Qian, S., Zhang, S. \& Zhang, Z. Single cell RNA sequencing of 13 human tissues identify cell types and receptors of human coronaviruses. Biochem. Biophys. Res. Commun. 526, 135-140 (2020).

135. Zhao, Y. et al. Single-cell RNA expression profiling of ACE2, the receptor of SARS-CoV-2. Am. J. Respir. Crit Care Med. 202, 756-759 (2020).

136. Lukassen, S. et al. SARS-CoV-2 receptor ACE2 and TMPRSS2 are primarily expressed in bronchial transient secretory cells. EMBO J. 39, e105114 (2020).

137. Laporte, M. \& Naesens, L. Airway proteases: an emerging drug target for influenza and other respiratory virus infections. Curr. Opin. Virol. 24 16-24 (2017).

138. Ou, T. et al. Hydroxychloroquine-mediated inhibition of SARS-CoV-2 entry is attenuated by TMPRSS2. PLoS Pathog. 17, e 1009212 (2021).

This study uses TMPRSS2 and cathepsin L inhibitors to show that SARS-CoV-2 is more dependent than SARS-COV on TMPRSS2.

139. Ozono, S. et al. SARS-CoV-2 D614G spike mutation increases entry efficiency with enhanced ACE2-binding affinity. Nat. Commun. 12, 848 (2021).

140. Zhu, Y. et al. A genome-wide CRISPR screen identifies host factors that regulate SARS-CoV-2 entry. Nat. Commun. 12, 961 (2021).

141. Hoffmann, M. et al. SARS-CoV-2 cell entry depends on ACE2 and TMPRSS2 and Is blocked by a clinically proven protease inhibitor. Cell 181, 271-280 (2020). This is the first study to confirm that processing of the SARS-CoV-2 $S$ protein is, like that of the SARS-CoV S protein, mediated by TMPRSS2

142. Bosch, B. J., Bartelink, W. \& Rottier, P. J. Cathepsin L functionally cleaves the severe acute respiratory syndrome coronavirus class I fusion protein upstream of rather than adjacent to the fusion peptide. J. Virol. 82, 8887-8890 (2008).

143. Ebert, D. H., Deussing, J., Peters, C. \& Dermody, T. S Cathepsin $L$ and cathepsin $B$ mediate reovirus 
disassembly in murine fibroblast cells. J. Biol. Chem. 277, 24609-24617 (2002).

144. Chandran, K., Sullivan, N. J., Felbor, U., Whelan, S. P. \& Cunningham, J. M. Endosomal proteolysis of the ebola virus glycoprotein is necessary for infection. Science 308, 1643 (2005).

145. Shaik, M. M. et al. Structural basis of coreceptor recognition by HIV- 1 envelope spike. Nature 565 , 318-323 (2019)

146. Campbell, G. R., To, R. K., Hanna, J. \& Spector, S. A. SARS-CoV-2, SARS-CoV-1, and HIV- 1 derived ssRNA sequences activate the NLRP3 inflammasome in human macrophages through a non-classical pathway. iScience 24, 102295 (2021)

147. Jangra, S. et al. Sterilizing immunity against SARS-CoV-2 infection in mice by a single-shot and lipid amphiphile imidazoquinoline TLR7/8 agonistadjuvanted recombinant spike protein vaccine. Angew Chem. Int. Ed. 60, 9467-9473 (2021).

148. Shi, G. et al. Opposing activities of IFITM proteins in SARS-CoV-2 infection. EMBO J. 40, e106501 (2021).

149. Winstone, $\mathrm{H}$. et al. The polybasic cleavage site in SARS-CoV-2 spike modulates viral sensitivity to type I interferon and IFITM2. J. Virol. https://doi.org/10.1128/ JVI.02422-20 (2021).

150. Pfaender, S. et al. LY6E impairs coronavirus fusion and confers immune control of viral disease. Nat. Microbiol. 5, 1330-1339 (2020).

151. Zhao, X. et al. LY6E restricts entry of human coronaviruses, including currently pandemic SARSCoV-2. J. Virol. https://doi.org/10.1128/JVI.00562-20 (2020).

152. Brass, A. L. et al. The IFITM proteins mediate cellular resistance to influenza A H1 N 1 virus, West Nile virus, and dengue virus. Cell 139, 1243-1254 (2009).

153. Huang, I. C. et al. Distinct patterns of IFITM-mediated restriction of filoviruses, SARS coronavirus, and influenza A virus. PLoS Pathog. 7, e 1001258 (2011).

154. Liu, S. Y., Sanchez, D. J., Aliyari, R., Lu, S. \& Cheng, G. Systematic identification of type I and type II interferoninduced antiviral factors. Proc. Natl Acad. Sci. USA 109, 4239-4244 (2012).

155. Schoggins, J. W. et al. A diverse range of gene products are effectors of the type I interferon antiviral response. Nature 472, 481-485 (2011)

156. Yu, J., Liang, C. \& Liu, S. L. Interferon-inducible LY6E protein promotes HIV-1 infection. J. Biol. Chem. 292, 4674-4685 (2017)

157. Hackett, B. A. \& Cherry, S. Flavivirus internalization is regulated by a size-dependent endocytic pathway. Proc. Natl Acad. Sci. USA 115, 4246-4251 (2018)

158. Mar, K. B. et al. LY6E mediates an evolutionarily conserved enhancement of virus infection by targeting a late entry step. Nat. Commun. 9, 3603 (2018).

159. Li, X. et al. Emergence of SARS-CoV-2 through recombination and strong purifying selection. Sci. Adv. 6, eabb9153 (2020).

160. Boni, M. F. et al. Evolutionary origins of the SARS-CoV-2 sarbecovirus lineage responsible for the COVID-19 pandemic. Nat. Microbiol. 5, 1408-1417 (2020).

161. Menachery, V. D. et al. A SARS-like cluster of circulating bat coronaviruses shows potential for human emergence. Nat. Med. 21, 1508-1513 (2015)

162. Zhang, Y. Z. \& Holmes, E. C. A genomic perspective on the origin and emergence of SARS-CoV-2. Cell 181 223-227 (2020)

163. Andersen, K. G., Rambaut, A., Lipkin, W. I., Holmes, E. C. \& Garry, R. F. The proximal origin of SARS-CoV-2. Nat. Med. 26, 450-452 (2020).

164. Klimstra, W. B. et al. SARS-CoV-2 growth, furincleavage-site adaptation and neutralization using serum from acutely infected hospitalized COVID-19 patients. J. Gen. Virol. https://doi.org/10.1099/ jgv.0.001481 (2020)

165. Ogando, N. S. et al. SARS-coronavirus-2 replication in Vero E6 cells: replication kinetics, rapid adaptation and cytopathology. J. Gen. Virol. 101, 925-940 (2020).

166. Sasaki, M. et al. SARS-CoV-2 variants with mutations at the S1/S2 cleavage site are generated in vitro during propagation in TMPRSS2-deficient cells. PLoS Pathog. 17, e1009233 (2021).

This article shows that the furin-cleavage site in the SARS-CoV-2 S protein is rapidly lost during virus propagation in vitro, which was subsequently confirmed in multiple studies.

167. Pohl, M. O. et al. SARS-CoV-2 variants reveal features critical for replication in primary human cells. PLoS Biol. https://doi.org/10.1371/journal.pbio.3001006 (2021).

168. Mykytyn, A. Z. et al. SARS-CoV-2 entry into human airway organoids is serine protease-mediated and facilitated by the multibasic cleavage site. eLife https:// doi.org/10.7554/eLife. 64508 (2021)

169. Lamers, M. M. et al. Human airway cells prevent SARS-CoV-2 multibasic cleavage site cell culture adaptation. eLife 10, e66815 (2021)

170. Korber, B. et al. Tracking changes in SARS-CoV-2 spike: evidence that D614G increases infectivity of the COVID-19 virus. Cell 182, 812-827 (2020). This epidemiological study is first to raise the alarm about the rapid transmission of SARS-CoV-2 harbouring the $\mathrm{D} 614 \mathrm{G}$ mutation in the $\mathrm{S}$ protein

171. Jackson, C. B., Zhang, L., Farzan, M. \& Choe, H. Functional importance of the D614G mutation in the SARS-CoV-2 spike protein. Biochem. Biophys. Res. Commun. 538, 108-115 (2021)

172. Fernández, A. Structural impact of mutation D614C in SARS-CoV-2 spike protein: enhanced infectivity and therapeutic opportunity. ACS Med. Chem. Lett. 11, 1667-1670 (2020).

173. Michaud, W. A., Boland, G. M. \& Rabi, S. A. The SARS-CoV-2 spike mutation D614G increases entry fitness across a range of ACE2 levels, directly outcompetes the wild type, and is preferentially incorporated into trimers. Preprint at bioRxiv https:// doi.org/10.1101/2020.08.25.267500 (2020)

174. Juraszek, J. et al. Stabilizing the closed SARS-CoV-2 spike trimer. Nat. Commun. 12, 244 (2021).

175. Weissman, D. et al. D614G spike mutation increases SARS CoV-2 susceptibility to neutralization. Cell Host Microbe 29, 23-31 e24 (2021).

176. Yurkovetskiy, L. et al. Structural and functional analysis of the D614G SARS-CoV-2 spike protein variant. Cell https://doi.org/10.1016/j.cell.2020.09.032 (2020).

177. Benton, D. J. et al. The effect of the D614C substitution on the structure of the spike glycoprotein of SARS-CoV-2. Proc. Natl Acad. Sci. USA https:// doi.org/10.1073/pnas.2022586118 (2021).

178. Starr, T. N. et al. Deep mutational scanning of SARS $\mathrm{CoV}-2$ receptor binding domain reveals constraints on folding and ACE2 binding. Cell 182, 1295-1310 (2020).

179. Wong, S. K., Li, W., Moore, M. J., Choe, H. \& Farzan, M. A 193-amino acid fragment of the SARS coronavirus S protein efficiently binds angiotensinconverting enzyme 2. J. Biol. Chem. 279, 3197-3201 (2004).

This study is the first to identify the SARS-CoV RBD.

180. Liu, H. et al. The basis of a more contagious 501Y.V1 variant of SARS-CoV-2. Cell Res. 31, 720-722 (2021).

81. Tian, F. et al. N501Y mutation of spike protein in SARS-CoV-2 strengthens its binding to receptor ACE2. eLife https://doi.org/10.7554/eLife.69091 (2021).

182. $\mathrm{Gu}, \mathrm{H}$. et al. Adaptation of SARS-CoV-2 in BALB/c mice for testing vaccine efficacy. Science 369, 1603-1607 (2020).

183. Kidd, M. et al. S-variant SARS-CoV-2 lineage B1.1.7 is associated with significantly higher viral loads in samples tested by ThermoFisher TaqPath RT-qPCR J. Infect. Dis. https://doi.org/10.1093/infdis/jiab082 (2021).

184. Leung, K., Shum, M. H., Leung, G. M., Lam, T. T. $\&$ Wu, J. T. Early transmissibility assessment of the N501Y mutant strains of SARS-CoV-2 in the United Kingdom, October to November 2020. Euro Surveill. https://doi.org/10.2807/1560-7917.ES.2020.26.1. 2002106 (2021)

185. Yao, W. et al. Circulating SARS-CoV-2 variants B.1.1.7, 501 Y.V2 and P.1 have gained ability to utilize rat and mouse Ace 2 and altered in vitro sensitivity to neutralizing antibodies and ACE2-Ig. Preprint at bioRxiv https://doi.org/10.1101/2021.01.27.428353 (2021).

186. Montagutelli, X. et al. The B1.351 and P.1 variants extend SARS-CoV-2 host range to mice. Preprint at bioRxiv https://doi.org/10.1101/2021.03.18.436013 (2021).

187. Adam, D. What scientists know about new, fastspreading coronavirus variants. Nature 594, 19-20 (2021).

188. Frazier, L. et al. Spike protein cleavage-activation mediated by the SARS-CoV-2 P681R mutation: a casestudy from its first appearance in variant of interest (VOI) A.23.1 identified in Uganda. Preprint at bioRxiv https://doi.org/10.1101/2021.06.30.450632 (2021).

189. Hoffmann, M. et al. SARS-CoV-2 variants B. 1.351 and P. 1 escape from neutralizing antibodies. Cell 184 2384-2393 (2021).

190. Shen, X. et al. SARS-CoV-2 variant B.1.1.7 is susceptible to neutralizing antibodies elicited by ancestral spike vaccines. Cell Host Microbe https://doi.org/10.1016/ j.chom.2021.03.002 (2021)

191. Greaney, A. J. et al. Comprehensive mapping of mutations in the SARS-CoV-2 receptor-binding domain that affect recognition by polyclonal human plasma antibodies. Cell Host Microbe 29, 463-476 (2021)

192. Wang, P. et al. Increased resistance of SARS-CoV-2 variant P. 1 to antibody neutralization. Cell Host Microbe 29, 747-751 (2021).

193. Piccoli, L. et al. Mapping neutralizing and immunodominant sites on the SARS-CoV-2 spike receptor-binding domain by structure-guided highresolution serology. Cell 183, 1024-1042 (2020).

194. Thomson, E. C. et al. Circulating SARS-CoV-2 spike $\mathrm{N} 439 \mathrm{~K}$ variants maintain fitness while evading antibody-mediated immunity. Cell 184, 1171-1187 (2021).

195. Chen, R. E. et al. Resistance of SARS-CoV-2 variants to neutralization by monoclonal and serum-derived polyclonal antibodies. Nat. Med. 27, 717-726 (2021).

196. Baum, A. et al. Antibody cocktail to SARS-CoV-2 spike protein prevents rapid mutational escape seen with individual antibodies. Science 369, 1014-1018 (2020).

197. Starr, T. N. et al. Prospective mapping of viral mutations that escape antibodies used to treat COVID-19. Science 371, 850-854 (2021).

198. Andreano, E. et al. SARS-CoV-2 escape from a highly neutralizing COVID-19 convalescent plasma. Proc. Natl Acad. Sci. USA https://doi.org/10.1073/ pnas. 2103154118 (2021).

199. Deng, X. et al. Transmission, infectivity, and neutralization of a spike L452R SARS-CoV-2 variant. Cell 184, 3426-3437 (2021)

200. Cerutti, G. et al. Potent SARS-CoV-2 neutralizing antibodies directed against spike $\mathrm{N}$-terminal domain target a single supersite. Cell Host Microbe https:// doi.org/10.1016/j.chom.2021.03.005 (2021).

201. Lok, S. M. An NTD supersite of attack. Cell Host Microbe 29, 744-746 (2021)

202. McCallum, M. et al. N-terminal domain antigenic mapping reveals a site of vulnerability for SARS-CoV-2. Cell 184, 2332-2347 (2021).

203. Suryadevara, N. et al. Neutralizing and protective human monoclonal antibodies recognizing the $\mathrm{N}$-terminal domain of the SARS-CoV-2 spike protein Cell 184, 2316-2331 (2021).

204. Li, Q. et al. The impact of mutations in SARS-CoV-2 spike on viral infectivity and antigenicity. Cell 182, 1284-1294 (2020)

205. Weisblum, Y. et al. Escape from neutralizing antibodies by SARS-CoV-2 spike protein variants. eLife https:// doi.org/10.7554/eLife.61312 (2020).

206. Graham, R. L. \& Baric, R. S. Recombination, reservoirs, and the modular spike: mechanisms of coronavirus cross-species transmission. J. Virol. 84 3134-3146 (2010).

207. Frampton, D. et al. Genomic characteristics and clinical effect of the emergent SARS-CoV-2 B.1.1.7 lineage in London, UK: a whole-genome sequencing and hospital-based cohort study. Lancet Infect. Dis. https://doi.org/10.1016/s1473-3099(21)00170-5 (2021).

208. Davies, N. G. et al. Estimated transmissibility and impact of SARS-CoV-2 lineage B.1.1.7 in England Science 372, eabg3055 (2021).

209. Cele, S. et al. Escape of SARS-CoV-2 501Y.V2 from neutralization by convalescent plasma. Nature 593 . 142-146 (2021)

210. Shen, X. et al. Neutralization of SARS-CoV-2 Variants B. 1.429 and B.1.351. N. Engl. J. Med. https://doi.org 10.1056/NEJMc2103740 (2021).

211. Faria, N. R. et al. Genomics and epidemiology of the P. 1 SARS-CoV-2 lineage in Manaus, Brazil. Science 372, 815-821 (2021).

212. Vignier, N. et al. Breakthrough Infections of SARSCoV-2 Gamma variant in fully vaccinated gold miners, French Guiana, 2021. Emerg. Infect. Dis. https:// doi.org/10.3201/eid2710.211427 (2021).

213. Farinholt, T. et al. Transmission event of SARS-CoV-2 Delta variant reveals multiple vaccine breakthrough infections. Preprint at medRxiv https://doi.org/ 10.1101/2021.06.28.21258780 (2021).

214. Starr, T. N., Greaney, A. J., Dingens, A. S. \& Bloom, J. D. Complete map of SARS-CoV-2 RBD mutations that escape the monoclonal antibody LY-CoV555 and its cocktail with LY-CoV016. Cell Rep. Med. 2, 100255 (2021).

215. Zhou, H. et al. B. 1.526 SARS-CoV-2 variants identified in New York City are neutralized by vaccine-elicited and therapeutic monoclonal antibodies. Preprint at bioRxiv https://doi.org/10.1101/2021.03.24.436620 (2021).

216. Ferreira, I. et al. SARS-CoV-2 B.1.617 mutations L452 and $\mathrm{E} 484 \mathrm{Q}$ are not synergistic for antibody evasion. J. Infect. Dis. https://doi.org/10.1093/infdis/jiab368 (2021) 
217. Palacios, R. et al. Double-blind, randomized, placebocontrolled phase III clinical trial to evaluate the efficacy and safety of treating healthcare professionals with the adsorbed COVID-19 (inactivated) vaccine manufactured by Sinovac - PROFISCOV: a structured summary of a study protocol for a randomised controlled trial. Trials 21, 853 (2020).

218. Polack, F. P. et al. Safety and efficacy of the BNT $162 \mathrm{~b} 2$ mRNA Covid-19 vaccine. N. Engl. J. Med. 383 , 2603-2615 (2020)

219. Logunov, D. Y. et al. Safety and efficacy of an rAd26 and rAd5 vector-based heterologous prime-boost COVID-19 vaccine: an interim analysis of a randomised controlled phase 3 trial in Russia. Lancet 397, 671-681 (2021).

220. Baden, L. R. et al. Efficacy and safety of the mRNA1273 SARS-CoV-2 vaccine. N. Engl. J. Med. 384 403-416 (2021)

221. Barrett, J. R. et al. Phase $1 / 2$ trial of SARS-CoV-2 vaccine ChAdOx1 nCoV-19 with a booster dose induces multifunctional antibody responses. Nat. Med. 27, 279-288 (2021).

222. Ella, R. et al. Safety and immunogenicity of an inactivated SARS-CoV-2 vaccine, BBV152: a doubleblind, randomised, phase 1 trial. Lancet Infect. Dis. https://doi.org/10.1016/s1473-3099(20)30942-7 (2021).

223. Mercado, N. B. et al. Single-shot Ad26 vaccine protects against SARS-CoV-2 in rhesus macaques. Nature 586, 583-588 (2020).

224. Keech, C. et al. Phase 1-2 trial of a SARS-CoV-2 recombinant spike protein nanoparticle vaccine N. Engl. J. Med. 383, 2320-2332 (2020).

225. Gosert, R., Kanjanahaluethai, A., Egger, D., Bienz, K $\Sigma$ Baker, S. C. RNA replication of mouse hepatitis virus takes place at double-membrane vesicles. J. Virol. 76, 3697-3708 (2002).

226. Knoops, K. et al. SARS-coronavirus replication is supported by a reticulovesicular network of modified endoplasmic reticulum. PLoS Biol. 6, e226 (2008).

227. Snijder, E. J. et al. A unifying structural and functional model of the coronavirus replication organelle: tracking down RNA synthesis. PLoS Biol. 18, e3000715 (2020).

228. Stertz, S. et al. The intracellular sites of early replication and budding of SARS-coronavirus. Virology 361 , 304-315 (2007)

229. Wolff, G. et al. A molecular pore spans the double membrane of the coronavirus replication organelle. Science 369, 1395-1398 (2020).

230. Goldsmith, C. S. et al. Ultrastructural characterization of SARS coronavirus. Emerg. Infect. Dis. 10, 320-326 (2004).

231. Neuman, B. W. et al. A structural analysis of M protein in coronavirus assembly and morphology. J. Struct. Biol. 174, 11-22 (2011)

232. Schoeman, D. \& Fielding, B. C. Coronavirus envelope protein: current knowledge. Virol. J. 16, 69 (2019).

233. Beigel, J. H. et al. Remdesivir for the treatment of covid-19 - final report. N. Engl. J. Med. 383, 1813-1826 (2020)

234. US National Library of Medicine. ClinicalTrials.gov https://clinicaltrials.gov/ct2/show/NCT04321096 (2021).
235. Wang, P. G., Tang, D. J., Hua, Z., Wang, Z. \& An, J. Sunitinib reduces the infection of SARS-COV, MERS $\mathrm{COV}$ and SARS-CoV-2 partially by inhibiting AP2M1 phosphorylation. Cell Discov. 6, 71 (2020)

236. Zhang, Q. et al. Heparan sulfate assists SARS-CoV-2 in cell entry and can be targeted by approved drugs in vitro. Cell Discov. 6, 80 (2020).

237. Yang, Q. et al. Inhibition of SARS-CoV-2 viral entry upon blocking $\mathrm{N}$ - and O-glycan elaboration. eLife https://doi.org/10.7554/eLife.61552 (2020).

238. Zhu, Y, Yu, D., Yan, H., Chong, $\mathrm{H}$ \& \& He, Y Design of potent membrane fusion inhibitors against SARS $\mathrm{CoV}-2$, an emerging coronavirus with high fusogenic activity. J. Virol. 94, https://doi.org/10.1128/ JVI.00635-20 (2020).

239. Xiu, S. et al. Inhibitors of SARS-CoV-2 entry: current and future opportunities. J. Med. Chem. 63 , 12256-12274 (2020)

240. Salazar, E. et al. Treatment of coronavirus disease 2019 patients with convalescent plasma reveals a signal of significantly decreased mortality. Am. J. Pathol. 190, 2290-2303 (2020).

241. Libster, R. et al. Early high-titer plasma therapy to prevent severe Covid-19 in older adults. N. Engl. J. Med. 384, 610-618 (2021)

242. Li, L. et al. Effect of convalescent plasma therapy on time to clinical improvement in patients with severe and life-threatening COVID-19: a randomized clinical trial. JAMA 324, 460-470 (2020)

243. Liu, S. T. H. et al. Convalescent plasma treatment of severe COVID-19: a propensity score-matched control study. Nat. Med. 26, 1708-1713 (2020).

244. Simonovich, V. A. et al. A randomized trial of convalescent plasma in Covid-19 severe pneumonia. N. Engl. J. Med. 384, 619-629 (2021).

245. Joyner, M. J. et al. Convalescent plasma antibody levels and the risk of death from Covid-19. N. Engl. J. Med. 384, 1015-1027 (2021)

246. Wang, C. et al. A human monoclonal antibody blocking SARS-CoV-2 infection. Nat. Commun. 11, 2251 (2020).

247. Lv, Z. et al. Structural basis for neutralization of SARS-CoV-2 and SARS-CoV by a potent therapeutic antibody. Science 369, 1505-1509 (2020).

248. Koenig, P.-A. et al. Structure-guided multivalent nanobodies block SARS-CoV-2 infection and suppress mutational escape. Science 371, eabe6230 (2021).

249. Schoof, M. et al. An ultrapotent synthetic nanobody neutralizes SARS-CoV- 2 by stabilizing inactive spike. Science 370, 1473-1479 (2020).

250. Mahase, E. Covid-19: FDA authorises neutralising antibody bamlanivimab for non-admitted patients. BMJ 371, m4362 (2020)

251. Gottlieb, R. L. et al. Effect of bamlanivimab as monotherapy or in combination with etesevimab on viral load in patients with mild to moderate COVID-19: a randomized clinical trial. JAMA 325, 632-644 (2021).

252. Bhimraj, A. et al. Infectious Diseases Society of America Guidelines on the Treatment and Management of Patients with COVID-19 (Infectious Diseases Society of America, 2021).

253. Chen, Y. et al. ACE2-targeting monoclonal antibody as a "pan" coronavirus blocker in vitro and in a mouse model. Preprint at bioRxiv https://doi.org/10.1101/ 2020.11.11.375972 (2020)

254. Tada, T. et al. An ACE2 microbody containing a single immunoglobulin Fc domain is a potent inhibitor of SARS-CoV-2. Cell Rep. 33, 108528 (2020).

255. Monteil, V. et al. Inhibition of SARS-CoV-2 infections in engineered human tissues using clinical-grade soluble human ACE2. Cell 181, 905-913 (2020).

256. Sims, J. J. et al. Intranasal gene therapy to prevent infection by SARS-CoV-2 variants. PLoS Pathog. 17 e1009544 (2021)

257. Ferrari, M et al. Characterisation of a novel ACE2based therapeutic with enhanced rather than reduced activity against SARS-CoV-2 variants. J. Virol. https:// doi.org/10.1128/JVI.00685-21 (2021).

258. Haschke, M. et al. Pharmacokinetics and pharmacodynamics of recombinant human angiotensin-converting enzyme 2 in healthy human subjects. Clin. Pharmacokinet. 52, 783-792 (2013).

259. Khan, A. et al. A pilot clinical trial of recombinant human angiotensin-converting enzyme 2 in acute respiratory distress syndrome. Crit. Care https:/ doi.org/10.1186/s13054-017-1823-x (2017).

260. Reimer, J. M. et al. Matrix-M adjuvant induces local recruitment, activation and maturation of central immune cells in absence of antigen. PLOS ONE 7 , e41451 (2012).

261. Jackson, N. A. C., Kester, K. E., Casimiro, D. Gurunathan, S. \& DeRosa, F. The promise of mRNA vaccines: a biotech and industrial perspective. NPJ Vaccines https://doi.org/10.1038/s41541-020-0159-8 (2020).

\section{Acknowledgements}

This work was supported by an administrative supplement to NIH grant R01 Al1 29868 for severe acute respiratory syndrome coronavirus 2 (SARS-CoV-2) research to M.F. and H.C., a Fast grant by Emergent Ventures to B.C., a COVID-19 Award by the Massachusetts Consortium on Pathogen Readiness to B.C. and an administrative supplement to NIH grant Al147884 to B.C.

\section{Author contributions}

C.B.J., B.C. and H.C. researched data for the article. All authors contributed substantially to discussion of the content and wrote the article. C.B.J. and H.C. reviewed and edited the manuscript before submission.

\section{Competing interests}

The authors declare no competing interests.

\section{Peer review information}

Nature Reviews Molecular Cell Biology thanks A. Helenius, Y. Yamauchi and the other, anonymous, reviewer(s) for their contribution to the peer review of this work.

\section{Publisher's note}

Springer Nature remains neutral with regard to jurisdictional claims in published maps and institutional affiliations.

This is a U.S. government work and not under copyright protection in the U.S.; foreign copyright protection may apply. 2021 